\title{
Tight exponential analysis of universally composable privacy amplification and its applications
}

\author{
Masahito Hayashi Senior Member, IEEE
}

\begin{abstract}
Motivated by the desirability of universal composability, we analyze in terms of $L_{1}$ distinguishability the task of secret key generation from a joint random variable. Under this secrecy criterion, using the Rényi entropy of order $1+s$ for $s \in[0,1]$, we derive a new upper bound of Eve's distinguishability under the application of the universal ${ }_{2}$ hash functions. It is also shown that this bound gives the tight exponential rate of decrease in the case of independent and identical distributions. The result is applied to the wire-tap channel model and to secret key generation (distillation) by public discussion.
\end{abstract}

Index Terms-sacrifice bits, $L_{1}$ norm distance, universal composablity, secret key distillation, universal ${ }_{2}$ hash functions, wiretap channel

\section{INTRODUCTION}

Random privacy amplification based on the universal ${ }_{2}$ condition [1] has been studied by many authors [2], [3], [4], [5], [30], [6]. This technique is originally developed for random number extraction [2], [3]. It can also be applied to secret key generation (distillation) with public communication [7], [8], [9], [10], [11], [3], [4] and the wire-tap channel [12], [13], [14], [15], [16], [17], which treats the secure communication in the presence of an eavesdropper. (For details of its application, see e.g. the previous paper [6].) When random privacy amplification is implemented with universal ${ }_{2}$ hash functions, it can yield protocols for the above tasks with a relatively small amount of calculation.

Similar to the study [2], [30] for random privacy amplification based on the universal ${ }_{2}$ condition, the previous paper [6] focused only on the mutual information with the eavesdropper. However, as the secrecy criterion, many papers in the cryptography community [22], [3], [4], [5] adopt the half of the $L_{1}$ norm distance, so called $L_{1}$ distinguishability because this criterion is closely related to universally composable security [22]. In this paper, we adopt $L_{1}$ distinguishability as the secrecy criterion, and evaluate the secrecy for random privacy amplification. In the independent and identically distributed case, when the rate of generated random numbers is smaller than the entropy of the original information source, it is possible to generate a random variable whose $L_{1}$ norm distance to the uniform random number approaches zero asymptotically.

M. Hayashi was with Graduate School of Information Sciences, Tohoku University, Aoba-ku, Sendai, 980-8579, Japan and Centre for Quantum Technologies, National University of Singapore, 3 Science Drive 2, Singapore 117542. He is now with Graduate School of Mathematics, Nagoya University, Furocho, Chikusaku, Nagoya, 464-860, Japan and Centre for Quantum Technologies, National University of Singapore, 3 Science Drive 2, Singapore 117542. (e-mail: masahito@math.nagoya-u.ac.jp)
In the realistic setting, we can manipulate only a finite size of random variables. In order to treat the performance in the finite length setting, we have two kinds of formalism for the independent and identical distribution setting.

The first one is the second order formalism, in which, we focus on the asymptotic expansion up to second order in $\sqrt{n}$ of the length of the generated keys $l_{n}$ as $l_{n}=H n+C \sqrt{n}+o(\sqrt{n})$ with a constant constraint for the security parameter. The second one is the exponent formalism, in which, we fix the generation rate $R:=l_{n} / n$ and evaluate the exponential decreasing rate of convergence of the security parameter. In the exponent formalism, it is not sufficient to show that the security parameter goes zero exponentially, and it is required to explicitly give lower and/or upper bounds for the exponential decreasing rate. The exponent formalism has been studied by various information theoretical settings, e.g., channel coding [20], [35], source coding [19], [31], and mutual information criterion in wire-tap channel [17], [6]. As for the second order formalism, the optimal coding length with the fixed error probability has been derived up to the second order $\sqrt{n}$ in various settings [36], [37], [38] in the case of channel coding. In particular, the previous paper [37] treats it based on the information spectrum approach [32], which is closely related to $\epsilon$-smooth min-entropy. Note that, as is mentioned by Han [32], the information spectrum approach cannot yield the optimal exponent of error probability in the channel coding.

Concerning the second order formalism for uniform random number generation, the previous paper [25] has solved the optimal second order coefficient under $L_{1}$ distinguishability criterion and other criteria by employing the information spectrum method when there is no side information. Even when the side information exists, the same argument can be shown for the second order formalism by replacing the variance of the likelihood by the variance of the likelihood for the conditional distribution due to the following reason. For the converse part, the key lemma ([25, Lemma 4],[32, Lemma 2.1.2]) holds by replacing the distribution by the conditional distribution. The direct part can be shown by replacing the key lemma ([25, Lemma 3],[32, Lemma 2.1.1]) by the inequality (32) in the present paper, which holds under the universal ${ }_{2}$ hash functions.

However, the exponent formalism for $L_{1}$ distinguishability with secure key generation has not been studied sufficiently. Only the previous paper [6] treated it with mutual information criterion. Therefore, the present paper focuses on the exponent formalism for $L_{1}$ distinguishability. 
In Section [II] first, we focus on evaluation for random privacy amplification by Bennett et al [2], which employs the Rényi entropy of order 2. This evaluation was also obtained by Håstad et al [30] and is often called leftover hash lemma. Using a discussion similar to Renner [5], we derive an upper bound for the $L_{1}$ norm distance under the universal ${ }_{2}$ condition for hash functions, which is the main theorem of this paper (Theorem 11).

Next, we apply this theorem to the i.i.d. setting with a given key generation rate and a given source distribution. Then, we derive a lower bound of the exponent of the average of the $L_{1}$ norm distance between the generated random number and the uniform random number when a family of universal ${ }_{2}$ hash functions is applied. Next, we introduce a stronger condition for hash functions, which is called strongly universal ${ }_{2}$. We consider the $n$-independent and identical extension, and show that the exponential rate of decrease for this bound is tight under a stronger condition by using the type method, which was invented by Csiszár and Körner [19] and is one of standard methods in information theory. Since our bound realizes the optimal exponent, it gives a powerful bound even for the finite length setting [39]. One might consider that the smooth min entropy can derive the same lower bound for the exponential decreasing rate of universal composability. However, as shown in Subsection $\amalg I I-D$ the bound derived by the smooth min entropy is strictly smaller than that by smoothing of Rényi entropy of order 2. This disagreement is not so unnatural because a similar disagreement appears for the exponent of error probability in the channel coding as a relation between Gallager exponent and the lower bound derived by the information spectrum approach [32].

Further, if our protocol generating the random number is allowed to depend on the original distribution, there is a possibility to improve the exponent while it is known that asymptotic generation cannot be improved [26]. In Section IV] we derive the optimal exponent in this setting by using Cramér's Theorem [27] and the type method [19]. Based on comparison between this exponent and the exponent given in Section [III we can compare the performances between the protocol taking into account the full probability distribution of the source and the protocol based on the entropy of the source, which is realized by universal ${ }_{2}$ hash functions.

In Section V, we consider the case when an eavesdropper has a random variable correlated to the random variable of the authorized user. In this case, applying universal ${ }_{2}$ hash functions to his random variable, the authorized user obtain a secure random variable. We apply our evaluation of $L_{1}$ norm distance obtained in Subsection III-A (Theorem 11) to the distribution of the authorized user when the eavesdropper's random variable is fixed to a certain value. Then, we obtain a tighter evaluation (67) than that directly obtained from the previous paper [6].

In Section VI, we focus on wire-tap channel model, whose capacity has been calculated by Wyner [12] and Csiszár and Körner [13]. Csiszár [14] showed the strong security, and many papers [6], [33], [34] treat this model with mutual information criterion. The previous paper [17] derived bounds for both exponential rates of decrease for the security criterion based on the $L_{1}$ norm distance as well as the mutual information between Alice and Eve. It obtained a bound for the exponential rate of decrease concerning the $L_{1}$ security criterion. In this paper, we apply (67) to wire-tap channel model, and obtain the evaluation of the exponent of the $L_{1}$ security criterion. In Section VII it is shown that the evaluation obtained in this paper is better than that by the previous paper [17]. In a realistic setting, it is natural to restrict our codes to linear codes. In Section VIII, using (75), we provide a security analysis for a code constructed by the combination of an arbitrary linear code and privacy amplification by universal ${ }_{2}$ hash functions. This analysis yields the exponential rate of decrease for the $L_{1}$ security criterion. Overall, since (67) and (75) are derived from Theorem 1, all of the obtained results concerning the wire-tap channel model can be regarded as consequences of Theorem 1 .

Further, in Section $\amalg$, we obtain the bound for the $L_{1}$ security criterion in one-way secret key generation. In Appendix A] we prove Theorem 2 mentioned in Subsection [II-A. In Appendix B, we prove Lemma 6 given in Subsection [V] In Appendix $[$, we show Equation (37), which is important for comparison in Subsection $\amalg I I-D$.

\section{Relation with the previous paper [6]}

The main difference from the previous paper [6] is that the analysis on this paper is based on $L_{1}$ distinguishability while that on the previous paper [6] is based on the mutual information criterion. In the first step, this paper derives an evaluation (Theorem 11) of the equality of the uniform random number generation by universal ${ }_{2}$ hash functions based on the $L_{1}$ norm criterion. Applying Theorem 1 we treat several security problems. Since this paper treats the same security problems as the previous paper with the different criterion, some of protocols used in this paper were used in the previous paper [6]. That is, the coding protocols used in Sections VI] VIII and IX are used in Sections III, V, and VI in [6], respectively. While these protocols are described in [6], we describe the whole protocols in this paper for the readers' convenience.

For uniform random number generation, this paper gives the tight exponential rate of decrease for the $L_{1}$ norm distance, while the previous paper [6] gives a lower bound on the exponential rate of decrease based on Shannon entropy. Concerning secret key generation without communication, this paper gives a lower bound of the exponential rate of decrease based on $L_{1}$ distinguishability, while the previous paper [6] gives a lower bound of the exponential rate of decrease based on the mutual information criterion. Applying Pinsker's inequality (5), we can derive a lower bound of the exponential rate of decrease based on $L_{1}$ distinguishability from the lower bound in [6]. As is shown in Lemma 8 in Subsection $\overline{V-B}$, our lower bound is (strictly) better than combination of Pinsker's inequality and the lower bound by [6] (except for special cases). Note that application of Pinsker's inequality (5) or (6) yields the half of the lower bound of the exponent of the mutual information as a lower bound of the exponent of universal composability. Indeed, we give a numerical example in Fig. 3, in which our bound is strictly better than that by [6]. 
Concerning the wire-tap channel in a general framework, the code given in this paper is quite similar to that in the previous paper [6]. However, the evaluation method in this paper is different from that of the previous paper [6] because the analysis in this paper is based on $L_{1}$ distinguishability while that in the previous paper [6] is based on the mutual information. In this model, we can derive a lower bound for the exponential rate of decrease based on $L_{1}$ distinguishability by the combination of Pinsker's inequality (5) and the result in [6]. As is shown in Section VII] our lower bound is better than this lower bound from [6]. Section VIII treats a more realistic setting by using linear codes. Even in this setting, as is explained in Remark 1, our lower bound is strictly better than the lower bound by [6] (except for special cases mentioned in Lemma 8). The same observation can be applied to secret key generation by public communication, which is discussed in Section IX

\section{Preliminaries}

First, we briefly explain some notation and basic knowledge in information theory. In order to evaluate the difference between two distributions $P^{X}$ and $\tilde{P}^{X}$, we employ the following quantities: the $L_{1}$ distance (variational distance)

$$
d_{1}\left(P^{X}, \tilde{P}^{X}\right):=\sum_{x}\left|P^{X}(x)-\tilde{P}^{X}(x)\right|,
$$

the $L_{2}$ distance

$$
d_{2}\left(P^{X}, \tilde{P}^{X}\right):=\sqrt{\sum_{x}\left(P^{X}(x)-\tilde{P}^{X}(x)\right)^{2}},
$$

and the KL-divergence

$$
D\left(P^{X} \| \tilde{P}^{X}\right):=\sum_{x} P^{X}(x)\left(\log P^{X}(x)-\log \tilde{P}^{X}(x)\right),
$$

where log expresses the natural logarithm. These definitions can be extended when the total measure is less than 1 i.e., $\sum_{a} P^{A}(a) \leq 1$. In the following, we call such $P^{A}$ a subdistribution. This extension for sub-distributions is crucial for the later discussion.

When a joint distribution $P^{X, Y}$ is given, we have the following equation

$$
\begin{aligned}
& d_{1}\left(P^{X, Y}, \tilde{P}^{X} \times P^{Y}\right)=\sum_{x, y}\left|P^{X, Y}(x, y)-\tilde{P}^{X}(x) P^{Y}(y)\right| \\
= & \sum_{y} P^{Y}(y) \sum_{x}\left|P^{X \mid Y}(x \mid y)-\tilde{P}^{X}(x)\right| \\
= & \sum_{y} P^{Y}(y) d_{1}\left(P^{X \mid Y=y}, \tilde{P}^{X}\right) .
\end{aligned}
$$

When $P^{X}, \tilde{P}^{X}$ are normalized distributions, as a relation between the KL-divergence and the $L_{1}$ distance, the Pinsker's inequality

$$
\frac{1}{2} d_{1}\left(P^{X}, \tilde{P}^{X}\right)^{2} \leq D\left(P^{X} \| \tilde{P}^{X}\right)
$$

is known [19]. That is,

$$
-\log d_{1}\left(P^{X}, \tilde{P}^{X}\right) \geq \frac{-1}{2}\left(\log D\left(P^{X} \| \tilde{P}^{X}\right)+\log 2\right) .
$$

These relations will be helpful for later discussions.

\section{UNIFORM RANDOM NUMBER GENERATION}

\section{A. Protocol based on universal 2 hash function: Direct part}

Firstly, we consider the uniform random number generation problem from a biased random number $a \in \mathcal{A}$, which obeys a probability distribution $P^{A}$ for finite cardinality $|\mathcal{A}|$. There are two types of protocols for this problem. One is a protocol specialized for the given distribution $P^{A}$. The other is a universal protocol that does not depend on the given distribution $P^{A}$. The aim of this section is evaluate the performance of the latter setting. In the latter setting, our protocol is given by a function $f$ from $\mathcal{A}$ to $\mathcal{M}=\{1, \ldots, M\}$.

The quality of the random number obeying the subdistribution $P^{A}$ is evaluated by

$$
d_{1}\left(P^{A}\right):=d_{1}\left(P^{A}, P^{A}(\mathcal{A}) P_{\text {mix }}^{A}\right),
$$

where $P_{\text {mix }}^{A}$ is the uniform distribution on $\mathcal{A}$. We also use the Rényi entropy of order $1+s$ :

$$
H_{1+s}\left(A \mid P^{A}\right):=\frac{-1}{s} \log \sum_{a} P^{A}(a)^{1+s} .
$$

The $L_{2}$ distance is written by using the Rényi entropy of order 2 as follows.

$$
d_{2}\left(P^{A}, P^{A}(\mathcal{A}) P_{\text {mix }}^{A}\right)^{2}=e^{-H_{2}\left(A \mid P^{A}\right)}-\frac{P^{A}(\mathcal{A})^{2}}{|\mathcal{A}|} .
$$

Now, we focus on an ensemble of functions $f_{\mathbf{X}}$ from $\mathcal{A}$ to $\mathcal{M}=\{1, \ldots, M\}$, where $\mathbf{X}$ denotes a random variable describing the stochastic behavior of the function $f_{\mathbf{X}}$. In this case, we adopt on the following quantity as a criterion of the secrecy:

$$
\begin{aligned}
& \mathrm{E}_{\mathbf{X}} d_{1}\left(P^{f_{\mathbf{X}}(A)}\right)=\mathrm{E}_{\mathbf{X}} d_{1}\left(P^{f_{\mathbf{X}}(A)}, P^{A}(\mathcal{A}) P_{\operatorname{mix}}^{f_{\mathbf{X}}(A)}\right) \\
= & d_{1}\left(P^{B, \mathbf{X}}, P^{A}(\mathcal{A}) P_{\mathrm{mix}}^{B} \times P^{\mathbf{X}}\right),
\end{aligned}
$$

where $B$ is the random variable $f_{\mathbf{X}}(A)$ and the final equation follows from (4). Hence, when the expectation $\mathrm{E}_{\mathbf{X}} d_{1}\left(P^{f_{\mathbf{X}}(A)}\right)$ is sufficiently small, the random variable $f_{\mathbf{X}}(A)$ is almost independent of the side information $\mathbf{X}$. Then, the choice $f_{\mathbf{X}}$ can be communicated between Alice and Bob without revealing anything about $f(A)$.

An ensemble of hash functions $f_{\mathbf{X}}$ is called universal ${ }_{2}$ when it satisfies the following condition [1]:

Condition 1 (Universal $)_{2}$ ): For any elements $a_{1} \neq a_{2} \in \mathcal{A}$, the collision probability that $f_{\mathbf{X}}\left(a_{1}\right)=f_{\mathbf{X}}\left(a_{2}\right)$ is at most $\frac{1}{M}$. We sometimes require the following additional condition:

Condition 2: For any $\mathbf{X}$, the cardinality of $f_{\mathbf{X}}^{-1}\{i\}$ does not depend on $i$.

This condition will be used in Section IV

Indeed, when the cardinality $|\mathcal{A}|$ is a power of a prime power $q$ and $M$ is another power of the same prime power $q$, as is shown in Appendix II of the previous paper [6], the ensemble $\left\{f_{\mathbf{X}}\right\}$ can be chosen to be the concatenation of a Toeplitz matrix and the identity $(\mathbf{X}, I)$ [18] only with $\log _{q}|\mathcal{A}|-1$ random variables taking values in the finite field $\mathbb{F}_{q}$. That is, the function can be obtained by the multiplication of the random matrix $(\mathbf{X}, I)$ taking values in $\mathbb{F}_{q}$. In this case, Condition 2 can be confirmed because the rank of $(\mathbf{X}, I)$ is constant. 
Bennett et al [2] essentially showed the following lemma. Lemma 1: A family of universal ${ }_{2}$ hash functions $f_{\mathbf{X}}$ satisfies

$$
\left.\mathrm{E}_{\mathbf{X}} e^{-H_{2}\left(f_{\mathbf{X}}(A) \mid P^{f} \mathbf{X}(A)\right.}\right) \leq e^{-H_{2}\left(A \mid P^{A}\right)}+\frac{P^{A}(\mathcal{A})^{2}}{M} .
$$

This was also shown by Håstad et al [30] and is often called leftover hash lemma.

Now, we follow the derivation of Theorem 5.5.1 of Renner [5] when one classical random variable is given. The Schwarz inequality implies that

$$
\begin{aligned}
& d_{1}\left(P^{f_{\mathbf{X}}(A)}, P^{A}(\mathcal{A}) P_{\text {mix }}^{f_{\mathbf{X}}(A)}\right) \\
\leq & \sqrt{M} \sqrt{d_{2}\left(P^{f_{\mathbf{X}}(A)}, P^{A}(\mathcal{A}) P_{\text {mix }}^{f_{\mathbf{X}}(A)}\right)} .
\end{aligned}
$$

Jensen's inequality yields that

$$
\begin{aligned}
& \mathrm{E}_{\mathbf{X}} d_{1}\left(P^{f_{\mathbf{X}}(A)}, P^{A}(\mathcal{A}) P_{\text {mix }}^{f_{\mathbf{X}}(A)}\right) \\
\leq & \sqrt{M} \sqrt{\mathrm{E}_{\mathbf{X}} d_{2}\left(P^{f_{\mathbf{X}}(A)}, P^{A}(\mathcal{A}) P_{\text {mix }}^{f_{\mathbf{X}}(A)}\right)} .
\end{aligned}
$$

Substituting (8) and (10) into the above inequality, we obtain

$$
\mathrm{E}_{\mathbf{X}} d_{1}\left(P^{f_{\mathbf{X}}(A)}\right) \leq M^{\frac{1}{2}} e^{-\frac{H_{2}(A \mid P A)}{2}} .
$$

Using (11), we can show the following theorem as a generalization of (11).

Theorem 1: A family of universal ${ }_{2}$ hash functions $f_{\mathbf{X}}$ satisfies

$$
\mathrm{E}_{\mathbf{X}} d_{1}\left(P^{f_{\mathbf{X}}(A)}\right) \leq 3 M^{\frac{s}{1+s}} e^{-\frac{s H_{1+s}\left(A \mid P^{A}\right)}{1+s}} \text { for } 0 \leq s \leq 1 .
$$

Substituting $s=1$, we obtain

$$
\mathrm{E}_{\mathbf{X}} d_{1}\left(P^{f \mathbf{X}(A)}\right) \leq 3 M^{\frac{1}{2}} e^{-\frac{H_{2}\left(A \mid P^{A}\right)}{2}} .
$$

Since the difference between (11) and (13) is only the coefficient, Theorem 1 can be regarded as a kind of generalization of Bennett et al [2]'s result [10].

Proof: For any $R^{\prime}>0$, we choose the subset $\Omega_{R^{\prime}}:=$ $\left\{P^{A}(a)>e^{-R^{\prime}}\right\}$, and define the sub-distribution $P_{R^{\prime}}^{A}$ by

Since

$$
P_{R^{\prime}}^{A}(a):= \begin{cases}0 & \text { if } a \in \Omega_{R^{\prime}} \\ P^{A}(a) & \text { otherwise. }\end{cases}
$$

$$
d_{1}\left(P^{A}, P_{R^{\prime}}^{A}\right)=P^{A}\left(\Omega_{R^{\prime}}\right)
$$

and

$$
\begin{aligned}
& d_{1}\left(P_{R^{\prime}}^{A}(\mathcal{A}) P_{\operatorname{mix}}^{f_{\mathbf{X}}(A)}, P^{A}(\mathcal{A}) P_{\operatorname{mix}}^{f_{\mathbf{X}}(A)}\right) \\
= & d_{1}\left(0,\left(P^{A}(\mathcal{A})-P_{R^{\prime}}^{A}(\mathcal{A})\right) P_{\operatorname{mix}}^{f_{\mathbf{X}}(A)}\right) \\
= & \left(P^{A}(\mathcal{A})-P_{R^{\prime}}^{A}(\mathcal{A})\right) d_{1}\left(0, P_{\operatorname{mix}}^{f_{\mathbf{X}}(A)}\right) \\
= & P^{A}(\mathcal{A})-P_{R^{\prime}}^{A}(\mathcal{A})=P^{A}\left(\Omega_{R^{\prime}}\right),
\end{aligned}
$$

the idea of "smoothing" by Renner [5] yields that

$$
\begin{aligned}
& d_{1}\left(P^{f_{\mathbf{X}}(A)}\right)=d_{1}\left(P^{f_{\mathbf{X}}(A)}, P^{A}(\mathcal{A}) P_{\operatorname{mix}}^{f_{\mathbf{X}}(A)}\right) \\
\leq & d_{1}\left(P^{f_{\mathbf{X}}(A)}, P_{R^{\prime}}^{f_{\mathbf{X}}(A)}\right)+d_{1}\left(P_{R^{\prime}}^{f_{\mathbf{X}}(A)}, P_{R^{\prime}}^{A}(\mathcal{A}) P_{\operatorname{mix}}^{f_{\mathbf{X}}(A)}\right) \\
& +d_{1}\left(P_{R^{\prime}}^{A}(\mathcal{A}) P_{\operatorname{mix}}^{f_{\mathbf{X}}(A)}, P^{A}(\mathcal{A}) P_{\operatorname{mix}}^{f_{\mathbf{X}}(A)}\right) \\
= & 2 P^{A}\left(\Omega_{R^{\prime}}\right)+d_{1}\left(P_{R^{\prime}}^{f_{\mathbf{X}}(A)}\right) .
\end{aligned}
$$

Taking the expectation over $\mathbf{X}$, we obtain

$$
\mathrm{E}_{\mathbf{X}} d_{1}\left(P^{f_{\mathbf{X}}(A)}\right) \leq 2 P^{A}\left(\Omega_{R^{\prime}}\right)+\mathrm{E}_{\mathbf{X}} d_{1}\left(P_{R^{\prime}}^{f_{\mathbf{X}}(A)}\right) .
$$

The inequality (11) yields

$$
\mathrm{E}_{\mathbf{X}} d_{1}\left(P_{R^{\prime}}^{f_{\mathbf{X}}(A)}\right) \leq M^{\frac{1}{2}} e^{-\frac{1}{2} H_{2}\left(A \mid P_{R^{\prime}}^{A}\right)} .
$$

For $0 \leq s \leq 1$, we can evaluate $e^{-H_{2}\left(A \mid P_{R^{\prime}}^{A}\right)}$ and $P^{A}\left(\Omega_{R^{\prime}}\right)$ as

$$
\begin{aligned}
& e^{-H_{2}\left(A \mid P_{R^{\prime}}^{A}\right)}=\sum_{a \in \Omega_{R^{\prime}}^{c}} P^{A}(a)^{2} \leq \sum_{a \in \Omega_{R^{\prime}}^{c}} P^{A}(a)^{1+s} e^{-(1-s) R^{\prime}} \\
\leq & \sum_{a} P^{A}(a)^{1+s} e^{-(1-s) R^{\prime}}=e^{-s H_{1+s}\left(A \mid P^{A}\right)-(1-s) R^{\prime}} \\
& P^{A}\left(\Omega_{R^{\prime}}\right)=\sum_{a \in \Omega_{R^{\prime}}} P^{A}(a) \leq \sum_{a \in \Omega_{R^{\prime}}}\left(P^{A}(a)\right)^{1+s} e^{s R^{\prime}} \\
\leq & \sum_{a}\left(P^{A}(a)\right)^{1+s} e^{s R^{\prime}}=e^{-s H_{1+s}\left(A \mid P^{A}\right)+s R^{\prime}}
\end{aligned}
$$

Combining (15), (16), and (17), for $R:=\log M$, we obtain

$$
\begin{aligned}
& \mathrm{E}_{\mathbf{X}} d_{1}\left(P^{f_{\mathbf{X}}(A)}\right) \\
\leq & 2 e^{-s H_{1+s}\left(A \mid P^{A}\right)+s R^{\prime}}+e^{R+\frac{1}{2}\left(-s H_{1+s}\left(A \mid P^{A}\right)-(1-s) R^{\prime}\right)} \\
= & 3 e^{-\frac{s H_{1+s}\left(A \mid P^{A}\right)+s R}{1+s}},
\end{aligned}
$$

where we substitute $\frac{R+s H_{1+s}\left(A \mid P^{A}\right)}{1+s}$ into $R^{\prime}$.

Next, we consider the case when our distribution $P^{A_{n}}$ is given by the $n$-fold independent and identical distribution of $P^{A}$, i.e, $\left(P^{A}\right)^{n}$. When the random number generation rate $\lim _{n \rightarrow \infty} \frac{1}{n} \log M_{n}$ is $R$, we focus on the exponential rate of decrease of $\mathrm{E}_{\mathbf{X}} d_{1}\left(P^{f_{\mathbf{X}, n}\left(A_{n}\right)}\right)$, and consider the supremum.

When an ensemble $\left\{f_{\mathbf{X}, n}\right\}$ of hash functions is a family of universal ${ }_{2}$ hash functions from $\mathcal{A}^{n}$ to $\left\{1, \ldots M_{n}\right\}$, Theorem 1 yields that

$$
\begin{aligned}
& \liminf _{n \rightarrow \infty} \frac{-1}{n} \log \mathrm{E}_{\mathbf{X}} d_{1}\left(P^{f_{\mathbf{X}, n}\left(A_{n}\right)}\right) \\
\geq & \frac{s H_{1+s}\left(A \mid P^{A}\right)-s R}{1+s}
\end{aligned}
$$

for $s \in[0,1]$. Taking the maximum over $s \in[0,1]$, we obtain

$$
\begin{aligned}
& \liminf _{n \rightarrow \infty} \frac{-1}{n} \log \mathrm{E}_{\mathbf{X}} d_{1}\left(P^{f_{\mathbf{X}, n}\left(A_{n}\right)}\right) \\
\geq & \max _{0 \leq s \leq 1} \frac{s H_{1+s}\left(A \mid P^{A}\right)-s R}{1+s} .
\end{aligned}
$$

On the other hand, when we apply the Pinsker's inequality [19] to the upper bound for the mutual information obtained by the previous paper [6], we obtain another bound $\max _{0 \leq s \leq 1} \frac{s H_{1+s}\left(A \mid P^{A}\right)-s R}{2}$, which is smaller than (18).

\section{B. Protocol based on universal 2 hash functions: Converse part}

In order to show the tightness of the exponential rate of decrease (18) under the universal ${ }_{2}$ condition, we consider the following property.

Condition 3 (Strongly universal ${ }_{2}$ ): For any $a \in \mathcal{A}$, $\operatorname{Pr}\left\{f_{\mathbf{X}}(a)=m\right\}=\frac{1}{M}$. The random variable $f_{\mathbf{X}}(a)$ is independent of $\left\{f_{\mathbf{X}}\left(a^{\prime}\right)\right\}_{a^{\prime}} \neq a \in \mathcal{A}$. 
Theorem 2: For any strongly universal 2 ensemble, any subset $\Omega \subset \mathcal{A}$ with $|\Omega|<M$ satisfies

$$
\mathrm{E}_{\mathbf{X}} d_{1}\left(P^{f_{\mathbf{X}}(A)}\right) \geq\left(1-\frac{|\Omega|}{M}\right)^{2} P^{A}(\Omega) .
$$

The proof is given in Appendix A

In order to derive the inequality opposite to (18) from Theorem 2] we employ the type method [19]. In the type method, when an $n$-trial data $\vec{a}_{n}:=\left(a_{1}, \ldots, a_{n}\right) \in \mathcal{A}^{n}$ is given, we focus on the distribution $p(a):=\frac{\#\left\{i \mid a_{i}=a\right\}}{n}$, which is called the empirical distribution for the data $\vec{a}_{n}$. In the type method, an empirical distribution is called a type. In the following, we denote the set of empirical distributions on $\mathcal{A}$ with $n$ trials by $\mathcal{T}_{n}$. The cardinality $\left|\mathcal{T}_{n}\right|$ is bounded by $(n+1)^{|\mathcal{A}|-1}$ [19], which increases polynomially with the number $n$. That is,

$$
\lim _{n \rightarrow \infty} \frac{1}{n} \log \left|\mathcal{T}_{n}\right|=0 \text {. }
$$

This property is the key idea in the type method. When $T_{n}(Q)$ represents the set of $n$-trial data whose empirical distribution is $Q$, the cardinality of $T_{n}(Q)$ can be evaluated as [19]:

$$
\left\lceil\frac{e^{n H(Q)}}{\left|\mathcal{T}_{n}\right|}\right\rceil \leq\left|T_{n}(Q)\right| \leq\left\lfloor e^{n H(Q)}\right\rfloor,
$$

where $\lceil x\rceil$ is the minimum integer $m$ satisfying $m \geq x$, and $\lfloor x\rfloor$ is the maximum $m$ satisfying $m \leq x$. Since any element $\vec{a} \in T_{n}(Q)$ satisfies

$$
P^{A_{n}}(\vec{a})=e^{-n\left(D\left(Q \| P^{A}\right)+H(Q)\right)},
$$

we obtain an important formula

$$
\frac{1}{\mathcal{T}_{n}} e^{-n D\left(Q \| P^{A}\right)} \leq P^{A_{n}}\left(T_{n}(Q)\right) \leq e^{-n D\left(Q \| P^{A}\right)} .
$$

Using the above knowledge, we can show the following proposition:

Proposition 1: When $M_{n}=\left\lfloor e^{n R}\right\rfloor$, any sequence of strongly universal ${ }_{2}$ ensembles $\left\{f_{\mathbf{X}, n}\right\}$ from $\mathcal{A}^{n}$ to $\left\{1, \ldots M_{n}\right\}$ satisfies the equation

$$
\limsup _{n \rightarrow \infty} \frac{-1}{n} \log \mathrm{E}_{\mathbf{X}} d_{1}\left(P^{f_{\mathbf{X}, n}\left(A_{n}\right)}\right) \leq \min _{Q: H(Q) \leq R} D\left(Q \| P^{A}\right),
$$

where $D\left(Q \| P^{A}\right)$ is the Kullback-Leibler divergence $\sum_{a \in \mathcal{A}} Q(a)\left(\log Q(a)-\log P^{A}(a)\right)$.

Proof: Choose an arbitrary empirical distribution $Q \in \mathcal{T}_{n}$ satisfying that $H(Q) \leq R$. Then, due to (21), the cardinality $\left|T_{n}(Q)\right|$ is less than $\left\lfloor e^{n R}\right\rfloor$. We choose the subset $\Omega_{n, Q}$ with the cardinality $\left\lceil\frac{1}{2} e^{n R}\right\rceil$ so that it contains at least $\left\lceil\frac{\left|T_{n}(Q)\right|}{2}\right\rceil$ elements of $T_{n}(Q)$. Using (21) and (22), we obtain

$$
\begin{aligned}
P^{A_{n}}\left(\Omega_{n, Q}\right) & \geq \frac{\left|T_{n}(Q)\right|}{2} e^{-n\left(D\left(Q \| P^{A}\right)+H(Q)\right)} \\
& \geq \frac{e^{n H(Q)}}{2\left|\mathcal{T}_{n}\right|} e^{-n\left(D\left(Q \| P^{A}\right)+H(Q)\right)} .
\end{aligned}
$$

Using Theorem 2 with $\Omega_{n, Q}$, we obtain

$$
\mathrm{E}_{\mathbf{X}} d_{1}\left(P^{f_{\mathbf{X}, n}\left(A_{n}\right)}\right) \geq\left(1-\frac{\left\lceil\frac{1}{2} e^{n R}\right\rceil}{\left\lfloor e^{n R}\right\rfloor}\right)^{2} \frac{1}{2\left|\mathcal{T}_{n}\right|} e^{-n D\left(Q \| P^{A}\right)} .
$$

Since $Q$ is an arbitrary empirical distribution $Q \in \mathcal{T}_{n}$ satisfying that $H(Q) \leq R$,

$$
\begin{aligned}
& \mathrm{E}_{\mathbf{X}} d_{1}\left(P^{f_{\mathbf{X}, n}\left(A_{n}\right)}\right) \\
\geq & \left(1-\frac{\left\lceil\frac{1}{2} e^{n R}\right\rceil}{\left\lfloor e^{n R}\right\rfloor}\right)^{2} \frac{1}{2\left|\mathcal{T}_{n}\right|} \max _{Q \in \mathcal{T}_{n}: H(Q) \leq R} e^{-n D\left(Q \| P^{A}\right)} .
\end{aligned}
$$

That is,

$$
\begin{aligned}
& \frac{-1}{n} \log \mathrm{E}_{\mathbf{X}} d_{1}\left(P^{f_{\mathbf{X}, n}\left(A_{n}\right)}\right) \\
& \leq_{Q \in \mathcal{T}_{n}: H(Q) \leq R} D\left(Q \| P^{A}\right)+\frac{1}{n} \log 2\left|\mathcal{T}_{n}\right| \\
& -\frac{2}{n} \log \left(1-\frac{\left\lceil\frac{1}{2} e^{n R}\right\rceil}{\left\lfloor e^{n R}\right\rfloor}\right) .
\end{aligned}
$$

Due to the continuity of $Q \mapsto H(Q), D\left(Q \| P^{A}\right)$ and (20), the limit $n \rightarrow \infty$ yields (24).

When $R \leq H\left(A \mid P^{A}\right)$, the equation

$$
\max _{0 \leq s} \frac{s\left(H_{1+s}\left(A \mid P^{A}\right)-R\right)}{1+s}=\min _{Q: H(Q) \leq R} D\left(Q \| P^{A}\right)
$$

is known as the strong converse exponent in fixed source coding [19], [31],[24, (A21)]. The maximum $\max _{0 \leq s} \frac{s\left(H_{1+s}\left(A \mid P^{A}\right)-R\right)}{1+s}$ is realized at $s=s_{0}$ when $R=$ $R_{s_{0}}:=\left.\left(1+s_{0}\right) \frac{d}{d s}\left(s H_{1+s}\left(A \mid P^{A}\right)\right)\right|_{s=s_{0}}-s_{0} H_{1+s_{0}}\left(A \mid P^{A}\right)$. Since $\frac{d}{d s} R_{s}=(1+s) \frac{d^{2}}{d s^{2}}\left(s H_{1+s}\left(A \mid P^{A}\right)\right) \leq 0, R_{s}$ is monotone decreasing with $s$.

Thus, when $H\left(A \mid P^{A}\right) \geq R \geq R_{1}$ ( $R_{1}$ is called the critical rate.),

$$
\max _{0 \leq s} \frac{s\left(H_{1+s}\left(A \mid P^{A}\right)-R\right)}{1+s}=\max _{0 \leq s \leq 1} \frac{s\left(H_{1+s}\left(A \mid P^{A}\right)-R\right)}{1+s} .
$$

Hence, in this case, due to (18), 24), (25), and (26), we obtain

$$
\begin{aligned}
& \lim _{n \rightarrow \infty} \frac{-1}{n} \log \mathrm{E}_{\mathbf{X}} d_{1}\left(P^{f_{\mathbf{X}, n}\left(A_{n}\right)}\right) \\
= & \max _{0 \leq s \leq 1} \frac{s\left(H_{1+s}\left(A \mid P^{A}\right)-R\right)}{1+s}=\min _{Q: H(Q) \leq R} D\left(Q \| P^{A}\right) .
\end{aligned}
$$

However, when $R<R_{1}$,

$$
\begin{aligned}
& \max _{0 \leq s \leq 1} \frac{s\left(H_{1+s}\left(A \mid P^{A}\right)-R\right)}{1+s}=\frac{H_{2}\left(A \mid P^{A}\right)-R}{2} \\
< & \max _{0 \leq s} \frac{s\left(H_{1+s}\left(A \mid P^{A}\right)-R\right)}{1+s} .
\end{aligned}
$$

So, the lower bound in (18) does not coincide with the upper bound in (24).

\section{Comparison with evaluation by Holenstein-Renner [29]}

In the above derivation, the key point is evaluating the probability $P^{A}\left(\Omega_{R^{\prime}}\right)$, which equals the probability $\left(P^{A}\right)^{n}\{a \in$ $\left.\mathcal{A}^{n} \mid\left(P^{A}\right)^{n}(a)>e^{-n R^{\prime}}\right\}$ in the $n$-i.i.d. setting. In the community of cryptography, the $n$-i.i.d. setting is not regarded as an important setting because they are more interested in the single-shot setting. In such a setting, they sometimes use Holenstein-Renner's [29] evaluation of $P^{X}\left(\Omega_{R^{\prime}}\right)$. They proved the following theorem. 
Theorem 3: When $0 \leq H(A)-R^{\prime} \leq \log |\mathcal{A}|$,

$$
\left(P^{A}\right)^{n}\left\{a \in \mathcal{A}^{n} \mid\left(P^{A}\right)^{n}(a)>e^{-n R^{\prime}}\right\} \leq 2^{-\frac{n\left(H(A)-R^{\prime}\right)^{2}}{2(\log (|\mathcal{A}|+3))^{2}}} .
$$

Further, when $|\mathcal{A}| \geq 3$ and $0 \leq H(A)-R^{\prime} \leq \frac{\log (|\mathcal{A}|-1)}{12}$,

$\left(P^{A}\right)^{n}\left\{a \in \mathcal{A}^{n} \mid\left(P^{A}\right)^{n}(a)>e^{-n R^{\prime}}\right\}>\frac{1}{110} 2^{-\frac{12 n\left(H(A)-R^{\prime}\right)^{2}}{(\log (|\mathcal{A}|-1))^{2}}}$.

When $|\mathcal{A}|=2$, the inequality yields the following evaluation. When $0 \leq H(A)-R^{\prime} \leq \frac{\log 3}{24}$,

$$
\left(P^{A}\right)^{n}\left\{a \in \mathcal{A}^{n} \mid\left(P^{A}\right)^{n}(a)>e^{-n R^{\prime}}\right\}>\frac{1}{110} 2^{-\frac{24 n\left(H(A)-R^{\prime}\right)^{2}}{(\log 3)^{2}}}
$$

for even $n$.

Our evaluation (17) of $\left(P^{A}\right)^{n}\left\{a \in \mathcal{A}^{n} \mid\left(P^{A}\right)^{n}(a)>e^{-n R^{\prime}}\right\}$ contains the parameter $0 \leq s \leq 1$. Since this parameter is arbitrary, it is natural to compare the upper bound $\min _{0 \leq s \leq 1} e^{-n\left(s H_{1+s}\left(X \mid P^{X}\right)-s R^{\prime}\right)}$ given by 177 with that by Theorem 3 . That is, using (17), we obtain the exponential evaluation

$$
\begin{aligned}
& \lim _{n \rightarrow \infty} \frac{-1}{n} \log \left(P^{A}\right)^{n}\left\{a \in \mathcal{A}^{n} \mid\left(P^{A}\right)^{n}(a)>e^{-n R^{\prime}}\right\} \\
\geq & \max _{0 \leq s} s H_{1+s}\left(A \mid P^{A}\right)-s R^{\prime},
\end{aligned}
$$

while Theorem 3 yields that

$$
\begin{aligned}
& \lim _{n \rightarrow \infty} \frac{-1}{n} \log \left(P^{A}\right)^{n}\left\{a \in \mathcal{A}^{n} \mid\left(P^{A}\right)^{n}(a)>e^{-n R^{\prime}}\right\} \\
\geq & \frac{\left(H(A)-R^{\prime}\right)^{2}}{2(\log (|\mathcal{A}|+3))^{2}} \log 2 .
\end{aligned}
$$

In this case, the upper bound is $\frac{12 \log 2\left(H(A)-R^{\prime}\right)^{2}}{(\log (|\mathcal{A}|-1))^{2}}$ for $|\mathcal{A}| \geq 3$ and $\frac{24 \log 2\left(H(A)-R^{\prime}\right)^{2}}{(\log 3)^{2}}$ for $|\mathcal{A}|=2$.

In fact, the probability $P^{A}\left(\Omega_{R^{\prime}}\right)$ is the key quantity in the method of information spectrum, which is a unified method in information theory [32]. When the method of information spectrum is applied to an i.i.d. source, the probability $P^{A}\left(\Omega_{R^{\prime}}\right)$ is evaluated by applying Cramér's Theorem (see [27]) to the random variable $\log P^{A}(a)$. Then we obtain

$$
\begin{aligned}
& \lim _{n \rightarrow \infty} \frac{-1}{n} \log \left(P^{A}\right)^{n}\left\{a \in \mathcal{A}^{n} \mid\left(P^{A}\right)^{n}(a)>e^{-n R^{\prime}}\right\} \\
= & \max _{0 \leq s} s H_{1+s}\left(A \mid P^{A}\right)-s R^{\prime}
\end{aligned}
$$

for $R \leq H(A)$. Since $s \mapsto s H_{1+s}\left(X \mid P^{X}\right)$ is concave, when $H(A) \geq R \geq H_{2}^{\prime}\left(A \mid P^{A}\right)$, the maximization (29) can be attained with $s \in[0,1]$, i.e.,

$$
\begin{aligned}
& \lim _{n \rightarrow \infty} \frac{-1}{n} \log \left(P^{A}\right)^{n}\left\{a \in \mathcal{A}^{n} \mid\left(P^{A}\right)^{n}(a)>e^{-n R^{\prime}}\right\} \\
= & \max _{0 \leq s \leq 1} s H_{1+s}\left(A \mid P^{A}\right)-s R^{\prime} .
\end{aligned}
$$

which implies that our evaluation (17) gives the tight bound for exponential rate of decrease for the probability $\left(P^{A}\right)^{n}\{a \in$ $\left.\mathcal{A}^{n} \mid\left(P^{A}\right)^{n}(a)>e^{-n R^{\prime}}\right\}$. In fact, the difference among these bounds is numerically given in Fig. 1. Therefore, we can conclude that our evaluation (17) is much better than that by Holenstein-Renner [29]. That is, the combination of Lemma 1 and (17) is essential for deriving the tight exponential bound.

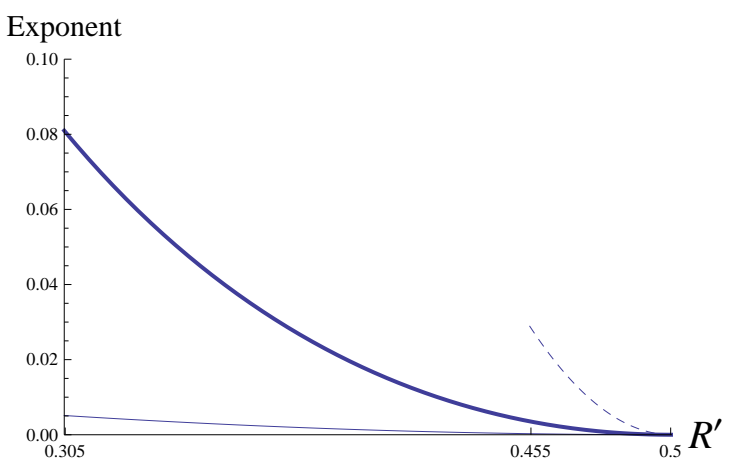

Fig. 1. Evaluation of $\lim _{n \rightarrow \infty} \frac{-1}{n} \log \left(P^{A}\right)^{n}\left\{a \in \mathcal{A}^{n} \mid\left(P^{A}\right)^{n}(a)>\right.$ $\left.e^{-n R^{\prime}}\right\}$. Thick line: $\max _{0 \leq s \leq 1} s H_{1+s}\left(A \mid P^{A}\right)-s R^{\prime}$ (The present paper), Normal line: $\frac{\left(H(A)-R^{\prime}\right)^{2}}{2(\log (|\mathcal{A}|+3))^{2}} \log 2$ (Lower bound by [29]), Dashed line: $\frac{24 \log 2\left(H(A)-R^{\prime}\right)^{2}}{(\log 3)^{2}}$ (Upper bound by [29]). Here, $P^{A}$ is chosen to be the binary distribution $P^{A}(0)=\alpha, P^{A}(1)=1-\alpha$ with $\alpha=0.200$. Then, $h(\alpha)=H(A)=0.500,\left.\frac{d\left(s H_{1+s}(A)\right)}{d s}\right|_{s=1}=0.305$, and $H(A)-\frac{\log 3}{24}=$ 0.455 .

\section{Comparison with smooth min-entropy}

In subsection III-A, we treated smoothing of Rényi entropy of order 2. In this subsection, we compare this method with smooth min-entropy, which is more familiar in the community of cryptography [5]. When we employ the minentropy $H_{\min }\left(A \mid P^{A}\right):=-\log \max _{a \in A} P^{A}(a)$ instead of Rényi entropy of order 2 in (11), we obtain the following inequality:

$$
\mathrm{E}_{\mathbf{X}} d_{1}\left(P^{f \mathbf{X}(A)}\right) \leq M^{\frac{1}{2}} e^{-\frac{H_{\min }\left(A \mid P^{A}\right)}{2}} .
$$

Now, we choose another distribution $\tilde{P}^{A}$ satisfying $d_{1}\left(\tilde{P}^{A}, P^{A}\right) \leq \epsilon$. Using (9), (30), and $\epsilon$-smooth minentropy $H_{\min , \epsilon}\left(A \mid P^{A}\right):=\max _{P: d_{1}\left(\tilde{P}^{A}, P^{A}\right) \leq \epsilon} H_{\min }\left(A \mid \tilde{P}^{A}\right)$, we can show the following inequality [5]

$$
\begin{aligned}
& \mathrm{E}_{\mathbf{X}} d_{1}\left(P^{f_{\mathbf{X}}(A)}\right)=\mathrm{E}_{\mathbf{X}} d_{1}\left(P^{f_{\mathbf{X}}(A)}, P^{A}(\mathcal{A}) P_{\operatorname{mix}}^{f_{\mathbf{X}}(A)}\right) \\
\leq & \mathrm{E}_{\mathbf{X}} d_{1}\left(\tilde{P}^{f_{\mathbf{X}}(A)}, \tilde{P}^{A}(\mathcal{A}) P_{\operatorname{mix}}^{f_{\mathbf{X}}(A)}\right)+d_{1}\left(\tilde{P}^{f_{\mathbf{X}}(A)}, P^{f_{\mathbf{X}}(A)}\right) \\
& +d_{1}\left(\tilde{P}^{A}(\mathcal{A}) P_{\operatorname{mix}}^{f_{\mathbf{X}}(A)}, P^{A}(\mathcal{A}) P_{\operatorname{mix}}^{f_{\mathbf{X}}(A)}\right) \\
\leq & M^{\frac{1}{2}} e^{-\frac{H_{\min }\left(A \mid \tilde{P}^{A}\right)}{2}}+d_{1}\left(\tilde{P}^{A}(A), P^{A}(A)\right) \\
& +\left|\tilde{P}^{A}(\mathcal{A}) P_{\operatorname{mix}}^{f_{\mathbf{X}}(A)}, P^{A}(\mathcal{A})\right| \\
\leq & M^{\frac{1}{2}} e^{-\frac{H_{\min , \epsilon}\left(A \mid P^{A}\right)}{2}}+2 \epsilon .
\end{aligned}
$$

Next, using the subdistribution $P_{R^{\prime}}^{A}$ defined in proof of Theorem 1, we choose $\epsilon$ to be $d_{1}\left(P^{A}, P_{R^{\prime}}^{A}\right)=P^{A}\left(\Omega_{R^{\prime}}\right)$ for a given $R^{\prime} \geq \log M$. Then,

$$
\begin{aligned}
& \mathrm{E}_{\mathbf{X}} d_{1}\left(P^{f_{\mathbf{X}}(A)}\right) \leq M^{\frac{1}{2}} e^{-\frac{H_{\min , \epsilon}\left(A \mid P^{A}\right)}{2}}+2 \epsilon \\
\leq & M^{\frac{1}{2}} e^{-\frac{H_{\min }\left(A \mid P_{R^{\prime}}^{A}\right)}{2}}+2 \epsilon \\
\leq & \sqrt{\frac{M}{e^{R^{\prime}}}}+2 P^{A}\left\{a \in \mathcal{A} \mid P^{A}(a)>e^{-R^{\prime}}\right\} .
\end{aligned}
$$

Applying the inequality (17), we obtain

$$
\mathrm{E}_{\mathbf{X}} d_{1}\left(P^{f_{\mathbf{X}}(A)}\right) \leq M^{\frac{1}{2}} e^{-R^{\prime} / 2}+2 e^{-s H_{1+s}\left(A \mid P^{A}\right)+s R^{\prime}}
$$


for $s \geq 0$. When $R=\log M$,

$$
\mathrm{E}_{\mathbf{X}} d_{1}\left(P^{f_{\mathbf{X}}(A)}\right) \leq e^{\left(R-R^{\prime}\right) / 2}+2 e^{-\left(s H_{1+s}\left(A \mid P^{A}\right)-s R^{\prime}\right)} .
$$

Now, we choose $R^{\prime}=R_{0}^{\prime}$ such that $\left(R_{0}^{\prime}-R\right) / 2=$ $s H_{1+s}\left(A \mid P^{A}\right)-s R_{0}^{\prime}$, which implies $R_{0}^{\prime}=\frac{R+2 s H_{1+s}\left(A \mid P^{A}\right)}{1+2 s}$. Hence, $\left(R_{0}^{\prime}-R\right) / 2=\frac{s H_{1+s}\left(A \mid P^{A}\right)-s R}{1+2 s}$. Thus, we obtain

$$
\mathrm{E}_{\mathbf{X}} d_{1}\left(P^{f_{\mathbf{X}}(A)}\right) \leq 3 e^{-\frac{s H_{1+s}\left(A \mid P^{A}\right)-s R}{1+2 s}}
$$

Taking the minimum over $s>0$, we have

$$
\mathrm{E}_{\mathbf{X}} d_{1}\left(P^{f_{\mathbf{X}}(A)}\right) \leq 3 e^{-\max _{s \geq 0} \frac{s H_{1+s}\left(A \mid P^{A}\right)-s R}{1+2 s}} .
$$

Next, we consider the case when our distribution $P^{A_{n}}$ is given by the $n$-fold independent and identical distribution of $P^{A}$, i.e, $\left(P^{A}\right)^{n}$. Similar to (18), 35 yields

$$
\begin{aligned}
& \liminf _{n \rightarrow \infty} \frac{-1}{n} \log \mathrm{E}_{\mathbf{X}} d_{1}\left(P^{f_{\mathbf{X}, n}\left(A_{n}\right)}\right) \\
\geq & \max _{0 \leq s} \frac{s H_{1+s}\left(A \mid P^{A}\right)-s R}{1+2 s} \\
= & \max _{0 \leq t \leq 1} \frac{t H_{1 /(1-t)}\left(A \mid P^{A}\right)-t R}{1+t},
\end{aligned}
$$

where $t=\frac{s}{1+s}$. In fact, as shown in Appendix $\mathrm{C}$, the exponential decreasing rate of the right hand side of (31) is calculated as

$$
\begin{aligned}
& \lim _{n \rightarrow \infty} \frac{-1}{n} \log \min _{\epsilon}\left(e^{\frac{n R}{2}} e^{-\frac{H_{\min , \epsilon}\left(A^{n} \mid\left(P^{A}\right)^{n}\right)}{2}}+2 \epsilon\right) \\
= & \max _{0 \leq t \leq 1} \frac{t H_{1 /(1-t)}\left(A \mid P^{A}\right)-t R}{1+t} .
\end{aligned}
$$

Hence, we can consider that $\max _{0 \leq t \leq 1} \frac{t H_{1 /(1-t)}\left(A \mid P^{A}\right)-t R}{1+t}$ expresses the optimal exponential decreasing rate for the method of smooth min-entropy. For $0 \leq t \leq 1$, the relation $t \leq$ $\frac{t}{1-t}$ implies the inequality $H_{1 /(1-t)}\left(A \mid P^{\bar{A}}\right) \leq H_{1+t}\left(A \mid P^{A}\right)$. Hence, the bound $\max _{0 \leq s} \frac{s H_{1+s}\left(A \mid P^{A}\right)-s R}{1+2 s}$ is smaller than the presented bound $\max _{0 \leq s \leq 1} \frac{s H_{1+s}\left(A \mid P^{A}\right)-s R}{1+s}$, whose numerical comparison is illustrated in Fig. 2 .

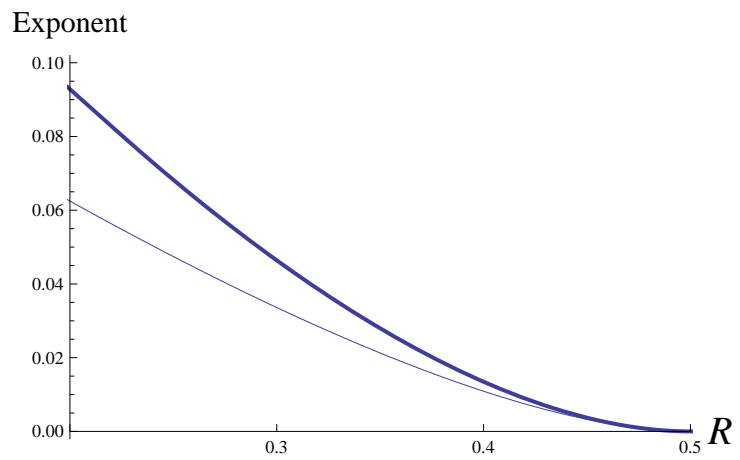

Fig. 2. Comparison between $\max _{0 \leq s} \frac{s H_{1+s}\left(A \mid P^{A}\right)-s R}{1+s}$ and $\max _{0 \leq s} \frac{s H_{1+s}\left(A \mid P^{A}\right)-s R}{1+2 s}$. Thick line: $\max _{0 \leq s} \frac{s H_{1+s}\left(A \mid P^{A}\right)-s R}{1+s}$ (Smoothing of Rényi entropy of order 2. The present paper), Normal line: $\max _{0 \leq s} \frac{s H_{1+s}\left(A \mid P^{A}\right)-s R}{1+2 s}$. (Smoothing of min entropy. [5]). Here, $P^{A}$ is chosen to be the binary distribution $P^{A}(0)=\alpha, P^{A}(1)=1-\alpha$ with $\alpha=0.200$. Then, $h(\alpha)=H(A)=0.500$.

\section{SPECIALIZED PROTOCOL FOR UNIFORM RANDOM NUMBER GENERATION}

\section{A. Main result of this section}

Next, we consider a function $f$ from $\mathcal{A}$ to $\{1, \ldots, M\}$ specialized to a given probability distribution $P^{A}$. This problem is called intrinsic randomness, which was studied with general source by Vembu and Verdú [26]. The previous paper [25] discussed the relation between the second order asymptotic rate and the central limit theorem. In the following, for the comparison with the exponential rate of decrease for (25), we prove the following theorem, which gives the optimal exponential rate of decrease for a given rate of uniform random number generation.

Theorem 4: When $\left.\frac{d\left(s H_{1+s}(A \mid P)\right)}{d s}\right|_{s=1} \leq R$, we obtain

$$
\begin{aligned}
& \lim _{n \rightarrow \infty} \frac{-1}{n} \log \min _{f_{n} \in \mathcal{F}_{n}(R)} d_{1}\left(P^{f_{n}\left(A_{n}\right)}\right) \\
= & \max _{0 \leq s \leq 1} s\left(H_{1+s}\left(A \mid P^{A}\right)-R\right),
\end{aligned}
$$

where $\mathcal{F}_{n}(R)$ is the set of functions $f_{n}$ from $\mathcal{A}^{n}$ to $\left\{1, \ldots,\left\lfloor e^{n R}\right\rfloor\right\}$.

Combining (27) and Theorem 4, we can compare the performances between a random universal protocol and the best specialized protocol. So, our exponential rate of decrease for the protocol based on universal ${ }_{2}$ hash functions is slightly smaller than the optimal exponential rate of decrease for specialized protocols.

In order to prove Theorem 4 we will show the following two inequalities:

$$
\begin{aligned}
& \limsup _{n \rightarrow \infty} \frac{-1}{n} \log \min _{f_{n} \in \mathcal{F}_{n}(R)} d_{1}\left(P^{f_{n}\left(A_{n}\right)}\right) \\
\leq & \max _{0 \leq s \leq 1} s\left(H_{1+s}\left(A \mid P^{A}\right)-R\right) \\
& \liminf _{n \rightarrow \infty} \frac{-1}{n} \log \min _{f_{n} \in \mathcal{F}_{n}(R)} d_{1}\left(P^{f_{n}\left(A_{n}\right)}\right) \\
\geq & \max _{0 \leq s \leq 1} s\left(H_{1+s}\left(A \mid P^{A}\right)-R\right) .
\end{aligned}
$$

Inequality (39) is called the converse part and Inequality (40) is called the direct part in the information theory community. In order to show the respective inequalities, we prepare respective lemmas (Lemmas 2 and (4) in the non-asymptotic setting in Subsection IV-B. In Subsection IV-C, using Lemma 4 and the concavity property, we show the converse part (39). Also, using Lemma 2, we show the direct part (39). In the latter derivation, we employ again the method of types [19].

\section{B. Non-asymptotic evaluation}

In order to treat the non-asymptotic case, we introduce the notation:

$$
[x]_{+}:= \begin{cases}x & \text { if } x \geq 0 \\ 0 & \text { if } x<0\end{cases}
$$

Then, the $L_{1}$ norm for two normalized distributions $P$ and $Q$ can be simplified to

$$
\sum_{a}|P(a)-Q(a)|=2 \sum_{a}[P(a)-Q(a)]_{+},
$$


which is a useful formula for the following discussion.

Hence, we obtain the following lemma, which is useful for our proof of the direct part (40).

Lemma 2: Any probability distribution $P^{A}$ and any function $f$ from $\mathcal{A}$ to $\{1, \ldots, M\}$ satisfy that

$$
d_{1}\left(P^{f(A)}\right) \geq P^{A}\left\{a \in \mathcal{A} \mid P^{A}(a) \geq \frac{2}{M}\right\} .
$$

Proof:

Any positive numbers $\alpha_{1}, \ldots, \alpha_{k}$ satisfies

$$
\left[\sum_{i=1}^{k} \alpha_{i}-\frac{1}{M}\right]_{+} \geq \sum_{i=1}^{k}\left[\alpha_{i}-\frac{1}{M}\right]_{+} .
$$

When $P^{A}(a) \geq \frac{2}{M}, P^{A}(a)-\frac{1}{M} \geq \frac{1}{M}$, which implies that

$$
\begin{aligned}
& 2\left[P^{A}(a)-\frac{1}{M}\right]_{+}=2\left(P^{A}(a)-\frac{1}{M}\right) \\
\geq & P^{A}(a)-\frac{1}{M}+\frac{1}{M}=P^{A}(a) .
\end{aligned}
$$

Thus, we obtain

$$
\begin{aligned}
& \sum_{b}\left|P^{A}\left(f^{-1}(b)\right)-\frac{1}{M}\right|=2 \sum_{b}\left[P^{A}\left(f^{-1}(b)\right)-\frac{1}{M}\right]_{+} \\
\geq & 2 \sum_{a \in \mathcal{A}}\left[P^{A}(a)-\frac{1}{M}\right]_{+} \\
\geq & 2 \sum_{a \in \mathcal{A}: P^{A}(a) \geq \frac{2}{M}}\left[P^{A}(a)-\frac{1}{M}\right]_{+} \\
\geq & \sum_{a \in \mathcal{A}: P^{A}(a) \geq \frac{2}{M}} P^{A}(a),
\end{aligned}
$$

where (45) and (46) follows from (43) and (44). Therefore, we obtain (42).

In order to show the converse part, we prepare the following lemma.

Lemma 3: Assume that for two integers $M \geq N$, two positive number sequences $\alpha_{1}, \ldots, \alpha_{N}$ and $\beta_{1}, \ldots, \beta_{M}$ satisfy that $\sum_{i=1}^{N} \alpha_{i} \geq \sum_{i=1}^{M} \beta_{i}$. Then, there exists a map $f$ from $\{1, \ldots, M\}$ to $\{1, \ldots, N\}$ such that

$$
\sum_{i=1}^{N}\left[\sum_{j \in f^{-1}(i)} \beta_{j}-\alpha_{i}\right]_{+} \leq N \max _{j} \beta_{j} .
$$

Proof: First, we define $f(1):=1$. For $j>1$, we define $f(j)$ inductively. When $\sum_{j^{\prime} \in f^{-1}(f(j-1))} \beta_{j^{\prime}}<\alpha_{f(j-1)}$, we define $f(j):=f(j-1)$. Otherwise, we define $f(j):=f(j-$ $1)+1$. Then the function satisfies the condition (47).

Now we consider the case when our distribution $P^{A_{n}}$ is given by the $n$-fold independent and identical distribution of $P^{A}$, i.e, $\left(P^{A}\right)^{n}$. Using Lemma 3, we have the following lemma, which is useful for our proof of the converse part (39).

Lemma 4: For any probability distribution $P^{A}$, there exists a function $f_{n}$ from $\mathcal{A}^{n}$ to $\left\{1, \ldots, M_{n}\right\}$ such that

$$
\begin{aligned}
& d_{1}\left(P^{f_{n}\left(A_{n}\right)}\right) \\
\leq & 2\left(P^{A}\right)^{n}\left\{a \in \mathcal{A}^{n} \mid\left(P^{A}\right)^{n}(a) \geq \frac{1}{M_{n}}\right\} \\
& +2 \sum_{Q \in \mathcal{T}_{n}^{1}\left[M_{n}\right]} M_{n} e^{-n\left(D\left(Q \| P^{A}\right)+H(Q)\right)} \cdot\left(P^{A}\right)^{n}\left(T_{n}(Q)\right) \\
& +2\left|\mathcal{T}_{n}\right| \max _{Q \in \mathcal{T}_{n}^{2}\left[M_{n}\right]} e^{-n\left(D\left(Q \| P^{A}\right)+H(Q)\right)}
\end{aligned}
$$

where

$$
\begin{aligned}
& \mathcal{T}_{n}^{1}\left[M_{n}\right]:=\left\{Q \in \mathcal{T}_{n} \mid D\left(Q \| P^{A}\right)+H(Q) \geq \frac{1}{n} \log M_{n}\right\} \\
& \mathcal{T}_{n}^{2}\left[M_{n}\right]:=\left\{Q \in \mathcal{T}_{n} \mid\left(P^{A}\right)^{n}\left(T_{n}(Q)\right)<\frac{1}{M_{n}}\right\} .
\end{aligned}
$$

Proof: In the first step, we define the function $f_{n}$. In the second step, we show that the function satisfies (48).

We divide $\mathcal{T}_{n}$ into three parts:

$$
\begin{aligned}
& \tilde{\mathcal{T}}_{n}^{0}\left[M_{n}\right]:=\left\{Q \in \mathcal{T}_{n} \mid e^{n\left(D\left(Q \| P^{A}\right)+H(Q)\right)} \leq M_{n}\right\} \\
& \tilde{\mathcal{T}}_{n}^{1}\left[M_{n}\right]:=\left\{Q \in\left(\tilde{\mathcal{T}}_{n}^{0}\left[M_{n}\right]\right)^{c} \cap \mathcal{T}_{n} \mid\left(P^{A}\right)^{n}\left(T_{n}(Q)\right) \geq \frac{1}{M_{n}}\right\} \\
& \tilde{\mathcal{T}}_{n}^{2}\left[M_{n}\right]:=\left\{Q \in\left(\tilde{\mathcal{T}}_{n}^{0}\left[M_{n}\right]\right)^{c} \cap \mathcal{T}_{n} \mid\left(P^{A}\right)^{n}\left(T_{n}(Q)\right)<\frac{1}{M_{n}}\right\},
\end{aligned}
$$

where $\left(\tilde{\mathcal{T}}_{n}^{0}\left[M_{n}\right]\right)^{c}$ is the complement of $\tilde{\mathcal{T}}_{n}^{0}\left[M_{n}\right]$. These three parts have the following relation with the above two parts:

$$
\tilde{\mathcal{T}}_{n}^{1}\left[M_{n}\right] \subset \mathcal{T}_{n}^{1}\left[M_{n}\right], \quad \tilde{\mathcal{T}}_{n}^{2}\left[M_{n}\right] \subset \mathcal{T}_{n}^{2}\left[M_{n}\right] .
$$

By using the integer $n_{Q}:=\left\lfloor\frac{\left(P^{A}\right)^{n}\left(T_{n}(Q)\right)}{1 / M_{n}}\right\rfloor=$ $\left\lfloor M_{n}\left(P^{A}\right)^{n}\left(T_{n}(Q)\right)\right\rfloor$, the conditions for $\tilde{\mathcal{T}}_{n}^{1}\left[M_{n}\right]$ and $\tilde{\mathcal{T}}_{n}^{2}\left[M_{n}\right]$ are written as $n_{Q} \geq 1$ and $n_{Q}<1$, respectively. Note that, since $n_{Q}$ is a non-negative integer, $n_{Q}<1$ is equivalent to $n_{Q}=0$.

Due to (22), the condition that $e^{n\left(D\left(Q \| P^{A}\right)+H(Q)\right)} \leq M_{n}$ is equivalent with the condition that $P^{A_{n}}(a) \geq \frac{1}{M_{n}}$ for $a \in$ $T_{n}(Q)$. Hence,

$$
\left(P^{A}\right)^{n}\left\{a \in \mathcal{A}^{n} \mid\left(P^{A}\right)^{n}(a) \geq \frac{1}{M_{n}}\right\}=\sum_{Q \in \mathcal{T}_{n}^{0}}\left(P^{A}\right)^{n}\left(T_{n}(Q)\right) .
$$

So,

$$
\begin{aligned}
& \left(P^{A}\right)^{n}\left\{a \in \mathcal{A}^{n} \mid\left(P^{A}\right)^{n}(a) \geq \frac{1}{M_{n}}\right\}+\sum_{Q \in \tilde{\mathcal{T}}_{n}^{1}\left[M_{n}\right]} \frac{n_{Q}}{M_{n}} \\
\leq & \sum_{Q \in \tilde{\mathcal{T}}_{n}^{0}\left[M_{n}\right]}\left(P^{A}\right)^{n}\left(T_{n}(Q)\right)+\sum_{Q \in \tilde{\mathcal{T}}_{n}^{1}\left[M_{n}\right]}\left(P^{A}\right)^{n}\left(T_{n}(Q)\right) \leq 1 .
\end{aligned}
$$

Since

$$
\frac{1}{M_{n}} \sum_{Q \in \tilde{\mathcal{T}}_{n}^{0}\left[M_{n}\right]}\left|T_{n}(Q)\right|=\frac{1}{M_{n}}\left|\left\{a \in \mathcal{A}^{n} \mid\left(P^{A}\right)^{n}(a) \geq \frac{1}{M_{n}}\right\}\right|
$$$$
\leq\left(P^{A}\right)^{n}\left\{a \in \mathcal{A}^{n} \mid\left(P^{A}\right)^{n}(a) \geq \frac{1}{M_{n}}\right\},
$$

we have

$$
\sum_{Q \in \tilde{\mathcal{T}}_{n}^{0}\left[M_{n}\right]}\left|T_{n}(Q)\right|+\sum_{Q \in \tilde{\mathcal{T}}_{n}^{1}\left[M_{n}\right]} n_{Q} \leq M_{n}
$$


Therefore, we can choose $f_{n}^{\prime}$ on $\Omega^{\prime} \quad:=$ $\cup_{Q \in \tilde{\mathcal{T}}_{n}^{0}\left[M_{n}\right] \cup \tilde{\mathcal{T}}_{n}^{1}\left[M_{n}\right]} T_{n}(Q)$ satisfying the following conditions.

1) For $Q, Q^{\prime} \in \tilde{\mathcal{T}}_{n}^{0}\left[M_{n}\right] \cup \tilde{\mathcal{T}}_{n}^{1}\left[M_{n}\right], \quad f_{n}^{\prime}\left(T_{n}(Q)\right) \cap$ $f_{n}^{\prime}\left(T\left(Q^{\prime}\right)\right)=\emptyset$.

2) $\left.f_{n}^{\prime}\right|_{T_{n}(Q)}$ is injective for $Q \in \tilde{\mathcal{T}}_{n}^{0}\left[M_{n}\right]$.

3) $\left|f_{n}^{\prime}\left(T_{n}(Q)\right)\right|=n_{Q}$ for $Q \in \tilde{\mathcal{T}}_{n}^{1}\left[M_{n}\right]$.

4) Any type $Q \in \tilde{\mathcal{T}}_{n}^{1}\left[M_{n}\right]$ satisfies that $\left|f_{n}^{\prime-1}(b)\right| \leq \frac{\left|T_{n}(Q)\right|}{n_{Q}}$ for $b \in f_{n}^{\prime}\left(T_{n}(Q)\right)$.

Then, for $Q \in \tilde{\mathcal{T}}_{n}^{1}\left[M_{n}\right]$, we obtain

$P^{f_{n}^{\prime}\left(A_{n}\right)}(b) \leq \frac{1}{M_{n}}+e^{-n\left(D\left(Q \| P^{A}\right)+H(Q)\right)}, \forall b \in f_{n}^{\prime}\left(T_{n}(Q)\right)$.

From the construction,

$$
\sum_{b \in f_{n}^{\prime}\left(\Omega^{\prime}\right)} P^{f_{n}^{\prime}\left(A_{n}\right)}(b) \geq \frac{1}{M_{n}}\left|f_{n}^{\prime}\left(\Omega^{\prime}\right)\right| .
$$

That is,

$$
\sum_{a \in\left(\Omega^{\prime}\right)^{c}} P^{A_{n}}(a) \leq \frac{1}{M_{n}}\left|\left(f_{n}^{\prime}\left(\Omega^{\prime}\right)\right)^{c}\right|
$$

Next, we define $f_{n}$ on the whole set by modifying $f_{n}^{\prime}$ as follows.

5) $f_{n}$ is the same as $f_{n}^{\prime}$ on $\Omega^{\prime}$.

6) Due to (51), we can apply Lemma 3 to the case when $\{1, \ldots, N\}=\left(f_{n}^{\prime}\left(\Omega^{\prime}\right)\right)^{c},\{1, \ldots, M\}=\left(\Omega^{\prime}\right)^{c}, \alpha_{b}=$ $\frac{1}{M_{n}}$ for $b \in\left(f_{n}^{\prime}\left(\Omega^{\prime}\right)\right)^{c}$ and $\beta_{a}=P^{A_{n}}(a)$ for $a \in\left(\Omega^{\prime}\right)^{c}$. Following Lemma 3 , we define the map $\left.f_{n}\right|_{\left(\Omega^{\prime}\right)^{c}}$ from $\left(\Omega^{\prime}\right)^{c}$ to $\left(f_{n}^{\prime}\left(\Omega^{\prime}\right)\right)^{c}$.

Our remaining task is to evaluate the value $\sum_{b}\left[P^{f_{n}\left(A_{n}\right)}(b)-\frac{1}{M_{n}}\right]_{+}$. Now, we define

$$
C(Q):=\sum_{b \in f_{n}\left(T_{n}(Q)\right)}\left[P^{f_{n}\left(A_{n}\right)}(b)-\frac{1}{M_{n}}\right]_{+} .
$$

Then, (49) implies that

$$
\sum_{Q \in \tilde{\mathcal{T}}_{n}^{0}\left[M_{n}\right]} C(Q) \leq\left(P^{A}\right)^{n}\left\{a \in \mathcal{A}^{n} \mid\left(P^{A}\right)^{n}(a) \geq \frac{1}{M_{n}}\right\} .
$$

For $Q \in \tilde{\mathcal{T}}_{n}^{1}\left[M_{n}\right]$, (50) implies

$$
\begin{aligned}
C(Q) & \leq n_{Q} e^{-n D\left(Q \| P^{A}\right)-n H(Q)} \\
& \leq M_{n} e^{-n D\left(Q \| P^{A}\right)-n H(Q)} \cdot\left(P^{A}\right)^{n}\left(T_{n}(Q)\right) .
\end{aligned}
$$

Thus, (52) and (53) imply

$$
\begin{aligned}
& \sum_{b \in f_{n}^{\prime}\left(\Omega^{\prime}\right)}\left[P^{f_{n}\left(A_{n}\right)}(b)-\frac{1}{M_{n}}\right]_{+} \\
\leq & \left(P^{A}\right)^{n}\left\{a \in \mathcal{A}^{n} \mid\left(P^{A}\right)^{n}(a) \geq \frac{1}{M_{n}}\right\} \\
& +\sum_{Q \in \mathcal{T}_{n}^{1}\left[M_{n}\right]} M_{n} e^{-n D\left(Q \| P^{A}\right)-n H(Q)} \cdot\left(P^{A}\right)^{n}\left(T_{n}(Q)\right) .
\end{aligned}
$$

Recall the condition 6). Lemma 3 guarantees that

$$
\begin{gathered}
\sum_{b \in\left(f_{n}^{\prime}\left(\Omega^{\prime}\right)\right)^{c}}\left[P^{f_{n}\left(A_{n}\right)}(b)-\frac{1}{M_{n}}\right]_{+} \\
\leq\left|\left(f_{n}^{\prime}\left(\Omega^{\prime}\right)\right)^{c}\right| \max _{Q \in \tilde{\mathcal{T}}_{n}^{2}\left[M_{n}\right]} e^{-n\left(D\left(Q \| P^{A}\right)+H(Q)\right)} \\
\leq\left|\mathcal{T}_{n}\right| \max _{Q \in \mathcal{T}_{n}^{2}\left[M_{n}\right]} e^{-n\left(D\left(Q \| P^{A}\right)+H(Q)\right)} .
\end{gathered}
$$

Combining (54) and (55), we obtain (48).

\section{Asymptotic evaluation}

Next, we proceed to the asymptotic evaluation. First, using Cramér's Theorem [27], we obtain

$$
\begin{aligned}
& \max _{0 \leq s} s H_{1+s}\left(A \mid P^{A}\right)-s R \\
= & \lim _{n \rightarrow \infty} \frac{-1}{n} \log \left(P^{A}\right)^{n}\left\{a \in \mathcal{A}^{n} \mid\left(P^{A}\right)^{n}(a) \geq \frac{1}{e^{n R}}\right\}
\end{aligned}
$$

Hence, Equality (56) and Lemma 2 imply

$$
\begin{aligned}
& \limsup _{n \rightarrow \infty} \frac{-1}{n} \log \min _{f_{n} \in \mathcal{F}_{n}(R)} d_{1}\left(P^{f_{n}\left(A_{n}\right)}\right) \\
\leq & \max _{0 \leq s} s\left(H_{1+s}\left(A \mid P^{A}\right)-R\right) .
\end{aligned}
$$

Since $\quad s \quad \mapsto \quad s H_{1+s}\left(A \mid P^{A}\right) \quad$ is concave, when $\left.\quad \frac{d\left(s H_{1+s}(A \mid P)\right)}{d s}\right|_{s=1} \leq R$, the maximum $\max _{0 \leq s} s\left(H_{1+s}\left(A \mid P^{A}\right)-R\right)$ is realized at $s \in[0,1]$, i.e., $\max _{0 \leq s \leq 1} s\left(H_{1+s}\left(A \mid P^{A}\right)-R\right)=\max _{0 \leq s} s\left(H_{1+s}\left(A \mid P^{A}\right)-\right.$ $R)$. Therefore, we obtain the converse part (39).

In order to show the direct part (40), we will show the following lemma by employing Lemma 2

Lemma 5:

$$
\begin{aligned}
& \liminf _{n \rightarrow \infty} \frac{-1}{n} \log \min _{f_{n} \in \mathcal{F}_{n}(R)} d_{1}\left(P^{f_{n}\left(A_{n}\right)}\right) \\
\geq & \max _{0 \leq s \leq 1} s\left(H_{1+s}\left(A \mid P^{A}\right)-R\right) .
\end{aligned}
$$

In order to show Lemma 5, we prepare the following lemma, whose proof is given in Appendix B

Lemma 6: When $\left.\frac{d\left(s H_{1+s}(A \mid P)\right)}{d s}\right|_{s=1} \leq R$,

$$
\begin{aligned}
& \min _{Q: H(Q)+D(Q \| P) \geq R} H(Q)+2 D(Q \| P)-R \\
= & \max _{0 \leq s} s H_{1+s}(A \mid P)-s R \\
= & \max _{0 \leq s \leq 1} s H_{1+s}(A \mid P)-s R .
\end{aligned}
$$

When $\left.\frac{d\left(s H_{1+s}(A \mid P)\right)}{d s}\right|_{s=1}>R$,

$$
\begin{aligned}
& \min _{Q: H(Q)+D(Q \| P) \geq R} H(Q)+2 D(Q \| P)-R \\
= & H_{2}(A \mid P)-R \\
= & \max _{0 \leq s \leq 1} s H_{1+s}(A \mid P)-s R .
\end{aligned}
$$


Proof of Lemma 5. Due to (20), (21), and the continuity of $Q \mapsto H(Q)$ and $D\left(Q \| P^{A}\right)$, we obtain

$$
\begin{aligned}
& \lim _{n \rightarrow \infty} \frac{-1}{n} \log 2\left|\mathcal{T}_{n}\right| \max _{Q \in \mathcal{T}_{n}^{2}\left[\left\lfloor e^{n R}\right\rfloor\right]} e^{-n\left(D\left(Q \| P^{A}\right)+H(Q)\right)} \\
= & \lim _{n \rightarrow \infty} \min _{Q \in \mathcal{T}_{n}^{2}\left[\left\lfloor e^{n R}\right\rfloor\right]} D\left(Q \| P^{A}\right)+H(Q) \\
= & \min _{Q: D\left(Q \| P^{A}\right) \geq R} D\left(Q \| P^{A}\right)+H(Q) \\
\geq & \min _{Q: D\left(Q \| P^{A}\right) \geq R} H(Q)+2 D\left(Q \| P^{A}\right)-R \\
\geq & \min _{Q: H(Q)+D\left(Q \| P^{A}\right) \geq R} H(Q)+2 D\left(Q \| P^{A}\right)-R .
\end{aligned}
$$

From (23),

$$
K_{n}:=\sum_{Q \in \mathcal{T}_{n}^{1}\left[\left\lfloor e^{n R}\right\rfloor\right]}\left\lfloor e^{n R}\right\rfloor\left(P^{A}\right)^{n}\left(T_{n}(Q)\right) e^{-n\left(D\left(Q \| P^{A}\right)+H(Q)\right)}
$$

satisfies that

$$
\begin{gathered}
\max _{Q \in \mathcal{T}_{n}^{1}\left[\left\lfloor e^{n R}\right\rfloor\right]} \frac{1}{\mathcal{T}_{n}} e^{-n\left(2 D\left(Q \| P^{A}\right)+H(Q)-R\right)} \\
\leq K_{n} \leq \mathcal{T}_{n} \max _{Q \in \mathcal{T}_{n}^{1}\left[\left\lfloor e^{n R}\right\rfloor\right]} e^{-n\left(2 D\left(Q \| P^{A}\right)+H(Q)-R\right)} .
\end{gathered}
$$

Due to (20) and the continuity of $Q \mapsto H(Q)$ and $D\left(Q \| P^{A}\right)$,

$$
\begin{aligned}
& \lim _{n \rightarrow \infty} \frac{-1}{n} \log K_{n} \\
& =\min _{Q: H(Q)+D\left(Q \| P^{A}\right) \geq R} H(Q)+2 D\left(Q \| P^{A}\right)-R .
\end{aligned}
$$

As is shown in Lemma 6 RHSs of (62) and (63) equal $\max _{0 \leq s \leq 1} s H_{1+s}\left(A \mid P^{A}\right)-s R$. Since $\max _{0 \leq s} s H_{1+s}\left(A \mid P^{A}\right)-s R \geq \max _{0 \leq s \leq 1} s H_{1+s}\left(A \mid P^{A}\right)-$ $s R$, (56) implies that

$$
\begin{aligned}
& \lim _{n \rightarrow \infty} \frac{-1}{n} \log \left(P^{A}\right)^{n}\left\{a \in \mathcal{A}^{n} \mid\left(P^{A}\right)^{n}(a) \geq \frac{2}{e^{n R}}\right\} \\
\geq & \max _{0 \leq s \leq 1} s\left(H_{1+s}\left(A \mid P^{A}\right)-R\right) .
\end{aligned}
$$

Thus, applying (62), 63), and 64) to the RHS of (48), and using Lemma 6, we can choose a sequence $\left\{f_{n}\right\}$ such that

$$
\begin{aligned}
& \liminf _{n \rightarrow \infty} \frac{-1}{n} \log \min _{f_{n}} d_{1}\left(P^{f_{n}\left(A_{n}\right)}\right) \\
\geq & \max _{0 \leq s \leq 1} s\left(H_{1+s}\left(A \mid P^{A}\right)-R\right),
\end{aligned}
$$

which implies (58).

\section{SECRET KEY GENERATION WITHOUT COMMUNICATION}

\section{A. Application of Theorem $\square$}

Next, we consider the secure key generation problem from a common random number $A \in \mathcal{A}$ which has been partially eavesdropped on by Eve. For this problem, it is assumed that Alice and Bob share a common random number $A \in \mathcal{A}$, and Eve has another random number $E \in \mathcal{E}$, which is correlated to the random number $A$. The task is to extract a common random number $f(A)$ from the random number $A \in \mathcal{A}$, which is almost independent of Eve's random number $E \in \mathcal{E}$. Here, Alice and Bob are only allowed to apply the same function $f$ to the common random number $A \in \mathcal{A}$.
Then, when the initial random variables $A$ and $E$ obey the distribution $P^{A, E}$, Eve's distinguishability can be represented by the following value:

$$
d_{1}\left(P^{f(A), E} \mid E\right):=d_{1}\left(P^{f(A), E}, P_{\operatorname{mix}}^{f(A)} \times P^{E}\right),
$$

where $P_{\text {mix }}^{f(A)} \times P^{E}$ is the product distribution of both marginal distributions $P_{\operatorname{mix}}^{f(A)}$ and $P^{E}$, and $P_{\operatorname{mix}}^{f(A)}$ is the uniform distribution on $\{1, \ldots, M\}$. While the half of this value directly gives the probability that Eve can distinguish Alice's information, we call $d_{1}\left(P^{f(A), E} \mid E\right)$ Eve's distinguishability in the following. This criterion was proposed by [22] and was used by [5]. Since the half of this quantity $d_{1}\left(P^{f(A), E} \mid E\right)$ is closely related to universally composable security, we adopt it as the secrecy criterion in this paper. As another criterion, we sometimes treat

$$
d_{1}^{\prime}\left(P^{f(A), E} \mid E\right):=d_{1}\left(P^{f(A), E}, P^{f(A)} \times P^{E}\right) .
$$

Since $d_{1}\left(P^{f(A)} \times P^{E}, P_{\text {mix }}^{M} \times P^{E}\right)=d_{1}\left(P^{f(A)}, P_{\text {mix }}^{M}\right) \leq$ $d_{1}\left(P^{f(A), E}, P_{\text {mix }}^{M} \times P^{E}\right)$, we have

$$
d_{1}^{\prime}\left(P^{f(A), E} \mid E\right) \leq 2 d_{1}\left(P^{f(A), E} \mid E\right) .
$$

Further, when $P^{f(A)}$ is the uniform distribution, the above criteria coincide with each other.

Next, we consider an ensemble of universal ${ }_{2}$ hash functions $\left\{f_{\mathbf{X}}\right\}$. Similar to (9), the equation

$$
\mathrm{E}_{\mathbf{X}} d_{1}\left(P^{f_{\mathbf{X}}(A), E} \mid E\right)=d_{1}\left(P^{B, E, \mathbf{X}}, P_{\mathrm{mix}}^{B} \times P^{E} \times P^{\mathbf{X}}\right)
$$

holds, where $B$ is the random variable $f_{\mathbf{X}}(A)$. Hence, when the expectation $\mathrm{E}_{\mathbf{X}} d_{1}\left(P^{f_{\mathbf{X}}(A), E} \mid E\right)$ is sufficiently small, the random variable $f_{\mathbf{X}}(A)$ is almost independent of the random variables $\mathbf{X}$ and $E$. So, the above value is suitable even when we randomly choose a hash function.

In order to evaluate the average performance, we define the quantity

$$
\begin{aligned}
\phi\left(t|A| E \mid P^{A, E}\right) & :=\log \sum_{e} P^{E}(e)\left(\sum_{a} P^{A \mid E}(a \mid e)^{\frac{1}{1-t}}\right)^{1-t} \\
& =\log \sum_{e}\left(\sum_{a} P^{A, E}(a, e)^{\frac{1}{1-t}}\right)^{1-t} .
\end{aligned}
$$

Note that when Eve's random variable $E$ takes a continuous value in the set $\mathcal{E}$, the relation 67 holds by defining $\phi\left(t|A| E \mid P^{A, E}\right)$ in the following way.

$$
\phi\left(t|A| E \mid P^{A, E}\right):=\log \int_{\mathcal{E}} P^{E}(e) d e\left(\sum_{a} P^{A \mid E}(a \mid e)^{\frac{1}{1-t}}\right)^{1-t} .
$$

This definition does not depend on the choice of the measure on $\mathcal{E}$.

By using Theorem 1 and putting $t=\frac{s}{1+s}$, any universal ${ }_{2}$ hash functions $\left\{f_{\mathbf{X}}\right\}$ satisfies the inequality:

$$
\begin{aligned}
\mathrm{E}_{\mathbf{X}} d_{1}\left(P^{f_{\mathbf{X}}(A), E} \mid E\right) & \leq 3 M^{\frac{s}{1+s}} \mathrm{E}_{e}\left(\sum_{a} P^{A \mid E}(a \mid e)^{1+s}\right)^{\frac{1}{1+s}} \\
& \left.=3 M^{t} e^{\phi\left(t|A| E \mid P^{A, E}\right.}\right)
\end{aligned}
$$

for $0 \leq t \leq \frac{1}{2}$. Therefore, there exists a function $f$ such that

$$
\begin{aligned}
d_{1}\left(P^{f(A), E} \mid E\right) & \leq 3 M^{\frac{s}{1+s}} \mathrm{E}_{e}\left(\sum_{a} P^{A \mid E}(a \mid e)^{1+s}\right)^{\frac{1}{1+s}} \\
& \left.=3 M^{t} e^{\phi\left(t \mid P^{A, E}\right.}\right)
\end{aligned}
$$


Next, we consider the case when our distribution $P^{A_{n} E_{n}}$ is given by the $n$-fold independent and identical distribution of $P^{A E}$, i.e, $\left(P^{A, E}\right)^{n}$. Ahlswede and Csiszár [7] showed that the optimal generation rate

$$
\begin{aligned}
& G\left(P^{A, E}\right) \\
:= & \sup _{\left\{\left(f_{n}, M_{n}\right)\right\}}\left\{\lim _{n \rightarrow \infty} \frac{\log M_{n}}{n} \mid \lim _{n \rightarrow \infty} d_{1}\left(P^{f_{n}\left(A_{n}\right), E_{n}} \mid E_{n}\right)=0\right\}
\end{aligned}
$$

equals the conditional entropy $H(A \mid E)$. That is, any achievable generation rate $R=\lim _{n \rightarrow \infty} \frac{\log M_{n}}{n}$ is no more than $H(A \mid E)$. The quantity $d_{1}\left(P^{f_{n}\left(A_{n}\right), E_{n}} \mid E_{n}\right)$ goes to zero. In order to treat the speed of this convergence, we focus on the supremum of the exponential rate of decrease (exponent) for $d_{1}\left(P^{f_{n}\left(A_{n}\right), E_{n}} \mid E_{n}\right)$ for a given $R$

$$
\begin{aligned}
& e_{1}\left(P^{A, E} \mid R\right) \\
&:=\sup _{\left\{\left(f_{n}, M_{n}\right)\right\}}\left\{\lim _{n \rightarrow \infty} \frac{-1}{n} \log d_{1}\left(P^{f_{n}\left(A_{n}\right), E_{n}} \mid E_{n}\right) \mid\right. \\
&\left.\qquad \lim _{n \rightarrow \infty} \frac{-1}{n} \log M_{n} \leq R\right\} .
\end{aligned}
$$

Since the relation $\phi\left(t\left|A^{n}\right| E^{n} \mid\left(P^{A, E}\right)^{n}\right)=n \phi\left(t|A| E \mid P^{A, E}\right)$ holds, the inequality (68) implies that

$$
e_{1}\left(P^{A, E} \mid R\right) \geq-\phi\left(t|A| E \mid P^{A, E}\right)-t R .
$$

for $t \in[0,1 / 2]$. That is, taking the maximum concerning $t \in$ $[0,1 / 2]$, we obtain

$$
e_{1}\left(P^{A, E} \mid R\right) \geq e_{\phi}\left(A|E| P^{A, E} \mid R\right),
$$

where

$$
\begin{aligned}
e_{\phi}\left(A|E| P^{A, E} \mid R\right) & :=\max _{0 \leq t \leq \frac{1}{2}}-\phi\left(t|A| E \mid P^{A, E}\right)-t R \\
& =\max _{0 \leq s \leq 1}-\phi\left(\frac{s}{1+s}|A| E \mid P^{A, E}\right)-\frac{s}{1+s} R .
\end{aligned}
$$

Since $\left.\frac{d}{d t} \phi\left(t \mid P^{A, E}\right)\right|_{t=0}=\left.\frac{d\left(s H_{1+s}\left(A|E| P^{A, E}\right)\right.}{d s}\right|_{s=0}=$ $-H(A \mid E)$, the right hand sides of (70) and (71) are strictly greater than 1 for $R<H(A \mid E)$.

\section{B. Comparison with the previous paper [6]}

Next, we show how better our bound is than that by the previous paper [6]. The previous paper [6] shows the following in Section IIA: there exists a sequence of functions $f_{n}: \mathcal{A}^{n} \rightarrow$ $\left\{1, \ldots,\left\lfloor e^{n R}\right\rfloor\right\}$ such that

$$
\begin{aligned}
& \lim _{n \rightarrow \infty} \frac{-1}{n} \log D\left(P^{f_{n}\left(A_{n}\right), E_{n}} \| P_{\operatorname{mix}}^{f_{n}\left(A_{n}\right)} \times P^{E_{n}}\right) \\
\geq & \max _{0 \leq s \leq 1} s H_{1+s}\left(A|E| P^{A, E}\right)-s R,
\end{aligned}
$$

where we define the function

$$
\begin{aligned}
s H_{1+s}\left(A|E| P^{A, E}\right) & :=-\log \sum_{a, e} P^{E}(e) P^{A \mid E}(a \mid e)^{1+s} \\
& =-\log \sum_{a, e} P^{A, E}(a, e)^{1+s} P^{E}(e)^{-s}
\end{aligned}
$$

for $s \in[0,1]$. Hence, applying Pinsker's inequality (6), we obtain

$$
\begin{aligned}
e_{1}\left(P^{A, E} \mid R\right) & \geq \lim _{n \rightarrow \infty} \frac{-1}{n} \log d_{1}\left(P^{f_{n}\left(A_{n}\right), E_{n}} \mid E_{n}\right) \\
& \geq \tilde{e}_{H}\left(A|E| P^{A, E} \mid R\right)
\end{aligned}
$$

where

$$
\begin{aligned}
\tilde{e}_{H}\left(A|E| P^{A, E} \mid R\right) & :=\max _{0 \leq s \leq 1} \frac{s H_{1+s}\left(A|E| P^{A, E}\right)-s R}{2} \\
& =\max _{0 \leq t \leq \frac{1}{2}} \frac{t H_{\frac{1}{1-t}}\left(A|E| P^{A, E}\right)-t R}{2-2 t}
\end{aligned}
$$

with $s=\frac{t}{1-t}$. Concerning the comparison of both bounds, we prepare the following lemma.

Lemma 7: The inequality

$$
-\frac{s}{1+s} H_{1+s}\left(A|E| P^{A, E}\right) \geq \phi\left(\frac{s}{1+s}|A| E \mid P^{A, E}\right)
$$

holds for $s \in(0, \infty)$. Equality holds if and only if the Rényi entropy $H_{1+s}\left(A \mid P^{A \mid E=e}\right)$ does not depend on the choice $e$ at the support of $P^{E}$.

Proof: Applying Jensen's inequality to the concave function $x \mapsto x^{\frac{1}{1+s}}$, we have

$$
\begin{aligned}
& e^{-\frac{s H_{1+s}\left(A|E| P^{A, E}\right)}{1+s}}=\left(\sum_{e} P^{E}(e) \sum_{a} P^{A \mid E}(a \mid e)^{1+s}\right)^{\frac{1}{1+s}} \\
\geq & \sum_{e} P^{E}(e)\left(\sum_{a} P^{A \mid E}(a \mid e)^{1+s}\right)^{\frac{1}{1+s}}=e^{\phi\left(\frac{s}{1+s}|A| E \mid P^{A, E}\right)} .
\end{aligned}
$$

Thus, the equality condition is that the value $\sum_{a} P^{A \mid E}(a \mid e)^{1+s}$ does not depend on the choice $e$ at the support of $P^{E}$. Hence, we obtain the desired argument.

In order to compare the two bounds $e_{\phi}\left(A|E| P^{A, E} \mid R\right)$ and $\tilde{e}_{H}\left(A|E| P^{A, E} \mid R\right)$, we introduce the following value:

$$
\begin{aligned}
e_{H}\left(A|E| P^{A, E} \mid R\right): & =\max _{0 \leq s \leq 1} \frac{s H_{1+s}\left(A|E| P^{A, E}\right)-s R}{1+s} \\
& =\max _{0 \leq t \leq \frac{1}{2}} t H_{\frac{1}{1-t}}\left(A|E| P^{A, E}\right)-t R
\end{aligned}
$$

Then, we obtain the following lemma.

Lemma 8:

$$
e_{\phi}\left(A|E| P^{A, E} \mid R\right) \geq e_{H}\left(A|E| P^{A, E} \mid R\right) \geq \tilde{e}_{H}\left(A|E| P^{A, E} \mid R\right)
$$

for $R<H(A \mid E)$. Equality in the first inequality holds if and only if the Rényi entropy $H_{1+s_{0}}\left(A \mid P^{A \mid E=e}\right)$ does not depend on the choice $e$ at the support of $P^{E}$ for $s_{0}:=$ $\operatorname{argmax}_{0 \leq s \leq 1}-\phi\left(\frac{s}{1+s}|A| E \mid P^{A, E}\right)-\frac{s}{1+s} R$. Equality in the second inequality holds if and only if $\frac{s H_{2}\left(A|E| P^{A, E}\right)-R}{2}=$ $\max _{0 \leq s \leq 1} \frac{s H_{1+s}\left(A|E| P^{A, E}\right)-s R}{1+s}$.

Therefore, our exponent $e_{\phi}\left(A|E| P^{A, E} \mid R\right)$ is strictly better than the exponent $\tilde{e}_{H}\left(A|E| P^{A, E} \mid R\right)$ by [6, Section IIA] except for the case satisfying the following two conditions: (i) $-\phi\left(\frac{1}{2}|A| E \mid P^{A, E}\right)-\frac{1}{2} R=\max _{0 \leq s \leq 1}-\phi\left(\frac{s}{1+s}|A| E \mid P^{A, E}\right)-$ $\frac{s}{1+s} R$. (ii) $H_{2}\left(A \mid P^{A \mid E=e}\right)$ does not depend on the choice $e$ at the support of $P^{E}$.

For example, we consider the following case: $\mathcal{A}$ equals $\mathcal{E}$, the set $\mathcal{A}$ has a module structure, (i.e., $\mathcal{A}$ is an Abelian 
group) and the conditional distribution $P^{A \mid E}(a \mid e)$ has the form $P^{A}(a-e)$. Then, the equality condition for the first inequality holds. Since

$$
\begin{aligned}
& e^{\phi\left(\frac{s}{1+s}|A| E \mid P^{A, E}\right)}=\sum_{e} P^{E}(e)\left(\sum_{a} P^{A \mid E}(a \mid e)^{1+s}\right)^{\frac{1}{1+s}} \\
= & \sum_{e} P^{E}(e) e^{-\frac{s H_{1+s}\left(A \mid P^{A}\right)}{1+s}}=e^{-\frac{s H_{1+s}\left(A \mid P^{A}\right)}{1+s}} .
\end{aligned}
$$

and

$$
\begin{aligned}
& e^{-s H_{1+s}\left(A|E| P^{A, E}\right)}=\sum_{e} P^{E}(e) \sum_{a} P^{A \mid E}(a \mid e)^{1+s} \\
= & \sum_{e} P^{E}(e) \sum_{a} P^{A}(a-e)^{1+s} \\
= & \sum_{e} P^{E}(e) e^{-\frac{s H_{1+s}\left(A \mid P^{A}\right)}{1+s}}=e^{-\frac{s H_{1+s}\left(A \mid P^{A}\right)}{1+s}}
\end{aligned}
$$

bounds $e_{\phi}\left(A|E| P^{A, E} \mid R\right)$ and $\tilde{e}_{H}\left(A|E| P^{A, E} \mid R\right)$ can be simplified to

$$
\begin{aligned}
e_{\phi}\left(A|E| P^{A, E} \mid R\right) & =e_{H}\left(A|E| P^{A, E} \mid R\right)=e_{H}\left(A\left|P^{A}\right| R\right) \\
\tilde{e}_{H}\left(A|E| P^{A, E} \mid R\right) & =\tilde{e}_{H}\left(A\left|P^{A}\right| R\right),
\end{aligned}
$$

where

$$
\begin{aligned}
e_{H}\left(A\left|P^{A}\right| R\right) & :=\max _{0 \leq s \leq 1} \frac{s H_{1+s}\left(A \mid P^{A}\right)-s R}{1+s} \\
& =\max _{0 \leq t \leq 1 / 2} t H_{\frac{1}{1-t}}\left(A \mid P^{A}\right)-t R \\
\tilde{e}_{H}\left(A\left|P^{A}\right| R\right) & :=\max _{0 \leq s \leq 1} \frac{s H_{1+s}\left(A \mid P^{A}\right)-s R}{2} \\
& =\max _{0 \leq t \leq 1 / 2} \frac{t H_{\frac{1}{1-t}}\left(A \mid P^{A}\right)-t R}{2-2 t} .
\end{aligned}
$$

In particular, both exponents are numerically plotted in Fig. 3 when $\mathcal{A}=\{0,1\}$, and $P^{A}(0)=a, P^{A}(1)=1-a$.

Proof: The first inequality and its equality condition follow from Lemma 7 and the definitions of $e_{\phi}\left(P^{A, E} \mid R\right)$ and $e_{H}\left(P^{A, E} \mid R\right)$. The second inequality follows from the inequality $\frac{1}{2} \leq \frac{1}{1+s}$ for $s \in[0,1]$. Since the equality holds only when $s=1$, we obtain the equality condition for the second inequality.

\section{THE WIRE-TAP CHANNEL IN A GENERAL FRAMEWORK}

Next, we consider the wire-tap channel model, in which the eavesdropper (wire-tapper) Eve and the authorized receiver Bob receive the information from the authorized sender Alice. In this case, in order for Eve to have less information, Alice chooses a suitable encoding. This problem is formulated as follows. Let $\mathcal{X}, \mathcal{Y}$ and $\mathcal{Z}$ be the alphabets of Alice, Bob, and Eve. Then, the main channel from Alice to Bob is described by $W^{B}: x \mapsto W_{x}^{B}$, and the wire-tapper channel from Alice to Eve is described by $W^{E}: x \mapsto W_{x}^{E}$. That is, $W_{x}^{B}$ is the output distribution on Bob's side with Alice's input $x$, and $W_{x}^{E}$ is the output distribution on Eve's side with Alice's input $x$. In this setting, in order to send a secret message in $\{1, \ldots, M\}$ subject to the uniform distribution, Alice chooses $M$ distributions $Q_{1}, \ldots, Q_{M}$ on $\mathcal{X}$, and she generates $x \in \mathcal{X}$ subject to $Q_{i}$ when she wants to send the

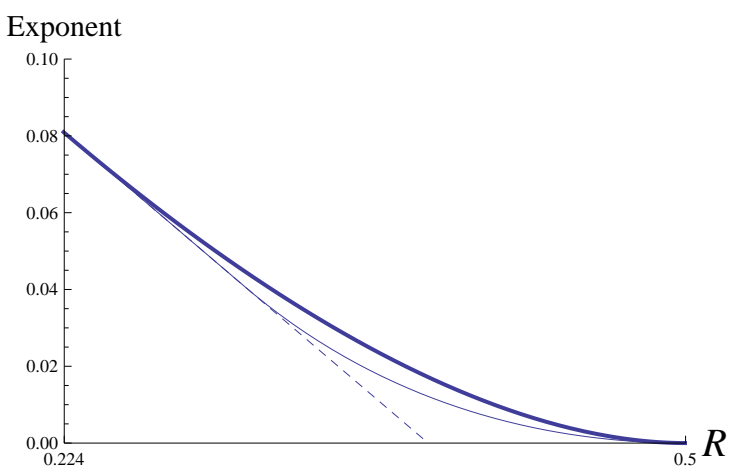

Fig. 3. Lower bounds of $e_{1}\left(P^{A E} \mid R\right)$. Thick line: $e_{H}\left(A\left|P^{A}\right| R\right)$ (The present paper), Normal line: $\tilde{e}_{H}\left(A\left|P^{A}\right| R\right)$ by [6]), Dashed line: $\frac{H_{2}\left(A \mid P^{A}\right)-s R}{2}$ (direct application of 111 without smoothing). Here, $P^{A}$ is chosen to be the binary distribution $P^{A}(0)=\alpha, P^{A}(1)=1-\alpha$ with $\alpha=0.200$. Then $h(\alpha)=H(A)=0.500$, and $\left.2 \frac{d\left(s H_{1+s}(A)\right)}{d s}\right|_{s=1}-$ $H_{2}(A)=0.224$

message $i \in\{1, \ldots, M\}$. Bob prepares $M$ disjoint subsets $\mathcal{D}_{1}, \ldots, \mathcal{D}_{M}$ of $\mathcal{Y}$ and judges that a message is $i$ if $y$ belongs to $\mathcal{D}_{i}$. Therefore, the triplet $\left(M,\left\{Q_{1}, \ldots, Q_{M}\right\},\left\{\mathcal{D}_{1}, \ldots, \mathcal{D}_{M}\right\}\right)$ is called a code, and is described by $\Phi$. Its performance is given by the following three quantities. The first is the size $M$, which is denoted by $|\Phi|$. The second is the average error probability $\epsilon_{B}(\Phi)$ :

$$
\epsilon_{B}(\Phi) \stackrel{\text { def }}{=} \frac{1}{M} \sum_{i=1}^{M} W_{Q_{i}}^{B}\left(\mathcal{D}_{i}^{c}\right),
$$

and the third is Eve's distinguishability $d_{1}(\Phi \mid E)$ :

$$
\begin{aligned}
d_{1}(\Phi \mid E) & :=d_{1}\left(W_{\Phi}^{E} \times P_{\mathrm{mix}}^{M}, W^{E}[\Phi]\right) \\
W_{\Phi}^{E}(e) & :=\sum_{i} \frac{1}{M} W_{Q_{i}}^{E}(e), \quad W^{E}[\Phi](i, e):=\frac{1}{M} W_{Q_{i}}^{E}(e) .
\end{aligned}
$$

The quantity $d_{1}(\Phi \mid E)$ gives an upper bound for the probability that Eve can succeed in distinguishing whether Alice's information belongs to a given subset. So, the value can be regarded as Eve's distinguishability. In order to calculate these values, we introduce the following quantity.

$$
\phi(t \mid W, p):=\log \sum_{y}\left(\sum_{x} p(x)\left(W_{x}(y)\right)^{1 /(1-t)}\right)^{1-t} .
$$

When the random variable $Y$ takes a continuous value in the set $\mathcal{Y}$ while $X$ takes discrete value, the above definition can be changed to

$$
\phi(t \mid W, p):=\log \int_{\mathcal{Y}}\left(\sum_{x} p(x)\left(W_{x}(y)\right)^{1 /(1-t)}\right)^{1-t} d y .
$$

This definition does not depend on the choice of the measure on $\mathcal{Y}$. That is, when $\tilde{W}_{x}(y) f(y)=W_{x}(y)$ for a positive function $f$,

$$
\phi(t \mid W, p)=\log \int_{\mathcal{Y}}\left(\sum_{x} p(x)\left(\tilde{W}_{x}(y)\right)^{1 /(1-t)}\right)^{1-t} f(y) d y .
$$


As is shown as Lemma 1 of [6], $\phi(t \mid W, p)$ satisfies the following lemma.

Lemma 9: The function $p \mapsto e^{\phi(t \mid W, p)}$ is convex for $t \in$ $[-1,0]$, and is concave for $t \in[0,1]$.

Now, using the function $\phi(t)$, we make a code for the wire-tap channel based on the random coding method. For this purpose, we make a protocol to share a random number. First, we generate the random code $\Phi(\mathbf{Y})$ with size $L M$, which is described as $\Phi(\mathbf{Y})(a)=Y_{a}$ for $a=1, \ldots, L M$ by using the $L M$ independent and identical random variables $\mathbf{Y}=\left(Y_{1}, \ldots, Y_{M L}\right)$ subject to the distribution $p$ on $\mathcal{X}$. Gallager [20] showed that the ensemble expectation of the average error probability concerning decoding the input message $A$ is less than $(M L)^{t} e^{\phi\left(-t \mid W^{B}, p\right)}$ for $0 \leq t \leq 1$ when Bob applies the maximum likelihood decoder $\mathcal{D}^{\prime}(\mathbf{Y})$ of the code $\Phi(\mathbf{Y})$. After sending the random variable $A$ taking values in the set with the cardinality $M L$, Alice and Bob apply the above universal ${ }_{2}$ hash functions $f_{\mathbf{X}}$ to the random variable $A$ and generate another piece of data of size $M$. Here, we assume that the ensemble $\left\{f_{\mathbf{X}}\right\}$ satisfies Condition 2, Then, Alice and Bob share the random variable $f_{\mathbf{X}}(A)$ with size $M$. This protocol is denoted by $\Phi(\mathbf{X}, \mathbf{Y})^{\prime}$.

Let $E$ be the random variable of the output of Eve's channel $W^{E}$. When $p$ is the uniform distribution on the set $\mathcal{C}:=\{1, \ldots, M L\}$ and the joint distribution $P^{C, E}$ is given by $P^{C, E}(c, e):=p(c) W_{c}^{E}(e)$, the equations

$$
\begin{aligned}
e^{\phi\left(t \mid P^{C, E}\right)} & =\frac{1}{M^{t} L^{t}} \sum_{e}\left(\sum_{a} p(c)\left(W_{c}^{E}(e)\right)^{\frac{1}{1-t}}\right)^{1-t} \\
& =\frac{e^{\phi\left(t \mid W^{E}, p\right)}}{M^{t} L^{t}}
\end{aligned}
$$

hold.

For a given code $\Phi(\mathbf{Y})$, we apply the inequality 67 to Eve's distinguishability. Then,

$$
\mathrm{E}_{\mathbf{X} \mid \mathbf{Y}} d_{1}\left(\Phi(\mathbf{X}, \mathbf{Y})^{\prime} \mid E\right) \leq 3 \frac{e^{\phi\left(t \mid W^{E}, p_{\text {mix }, \Phi(\mathbf{Y})}\right)}}{L^{t}}
$$

for $0 \leq t \leq \frac{1}{2}$. The concavity of $e^{\phi\left(t \mid W^{E}, p\right)}$ (Lemma 9) guarantees that

$$
\begin{aligned}
\mathrm{E}_{\mathbf{X}, \mathbf{Y}} d_{1}\left(\Phi(\mathbf{X}, \mathbf{Y})^{\prime} \mid E\right) & \leq 3 \mathrm{E}_{\mathbf{Y}} \frac{e^{\phi\left(t \mid W^{E}, p_{\mathrm{mix}, \Phi(\mathbf{Y})}\right)}}{L^{t}} \\
& \leq 3 \frac{e^{\phi\left(t \mid W^{E}, p\right)}}{L^{t}}
\end{aligned}
$$

for $0 \leq t \leq \frac{1}{2}$.

Now, we make a code for the wire-tap channel by modifying the above protocol $\Phi(\mathbf{X}, \mathbf{Y})^{\prime}$. First, we choose the distribution $Q_{i}$ to be the uniform distribution on $f_{\mathbf{X}}^{-1}\{i\}$. When Alice wants to send the secret message $i$, before sending the random variable $A$, Alice generates the random number $A$ subject to the distribution $Q_{i}$. Alice sends the random variable $A$. Bob recovers the random variable $A$ by using the maximum likelihood decoder $\mathcal{D}^{\prime}(\mathbf{Y})$, and applies the function $f_{\mathbf{X}}$. Then, Bob decodes Alice's message $i$, and this code for wire-tap channel $W^{B}, W^{E}$ is denoted by $\Phi(\mathbf{X}, \mathbf{Y})$. Since the ensemble $\left\{f_{\mathbf{X}}\right\}$ satisfies Condition 2 and the secret message $i$ obeys the uniform distribution on $\{1, \ldots, M\}$, this protocol $\Phi(\mathbf{X}, \mathbf{Y})$ has the same performance as the above protocol $\Phi(\mathbf{X}, \mathbf{Y})^{\prime}$.

Finally, we consider what code is derived from the above random coding discussion. Using the Markov inequality, we obtain

$$
\begin{array}{r}
\mathrm{P}_{\mathbf{X}, \mathbf{Y}}\left\{\epsilon_{B}(\Phi(\mathbf{X}, \mathbf{Y})) \leq 3 \mathrm{E}_{\mathbf{X}, \mathbf{Y}} \epsilon_{B}(\Phi(\mathbf{X}, \mathbf{Y}))\right\} \geq \frac{2}{3} \\
\mathrm{P}_{\mathbf{X}, \mathbf{Y}}\left\{d_{1}(\Phi(\mathbf{X}, \mathbf{Y}) \mid E) \leq 3 \mathrm{E}_{\mathbf{X}, \mathbf{Y}} d_{1}(\Phi(\mathbf{X}, \mathbf{Y}) \mid E)\right\} \geq \frac{2}{3} .
\end{array}
$$

Therefore, the existence of a good code is guaranteed in the following way. That is, we give the concrete performance of a code whose existence is shown in the above random coding method.

Theorem 5: There exists a code $\Phi$ for any integers $L, M$, and any probability distribution $p$ on $\mathcal{X}$ such that $|\Phi|=M$ and

$$
\begin{aligned}
\epsilon_{B}(\Phi) & \leq 3 \min _{0 \leq t \leq 1}(M L)^{t} e^{\phi\left(-t \mid W^{B}, p\right)}, \\
d_{1}(\Phi \mid E) & \leq 9 \min _{0 \leq t \leq \frac{1}{2}} \frac{e^{\phi\left(t \mid W^{E}, p\right)}}{L^{t}} .
\end{aligned}
$$

In the $n$-fold discrete memoryless channels $W^{B_{n}}$ and $W^{E_{n}}$ of the channels $W^{B}$ and $W^{E}$, the additive equation $\phi\left(t \mid W^{B_{n}}, p\right)=n \phi\left(t \mid W^{B}, p\right)$ holds. Thus, there exists a code $\Phi_{n}$ for any integers $L_{n}, M_{n}$, and any probability distribution $p$ on $\mathcal{X}$ such that $\left|\Phi_{n}\right|=M_{n}$ and

$$
\begin{aligned}
& \epsilon_{B}(\Phi) \leq 3 \min _{0 \leq t \leq 1}\left(M_{n} L_{n}\right)^{t} e^{n \phi\left(-t \mid W^{B}, p\right)}, \\
& d_{1}\left(\Phi_{n} \mid E\right) \leq 9 \min _{0 \leq t \leq \frac{1}{2}} \frac{e^{n \phi\left(t \mid W^{E}, p\right)}}{L_{n}^{t}} .
\end{aligned}
$$

Since $\lim _{t \rightarrow 0} \frac{\phi\left(t \mid W^{E}, p\right)}{t}=I\left(p: W^{E}\right)$, the rate $\max _{p} I(p$ : $\left.W^{B}\right)-I\left(p: W^{E}\right)^{t}$ can be asymptotically attained. Therefore, when the sacrifice information rate is $R$, i.e., $L_{n} \cong e^{n R}$, the exponential rate of decrease for Eve's distinguishability is greater than

$$
e_{\phi}\left(R \mid W^{E}, p\right):=\max _{0 \leq t \leq 1 / 2} t R-\phi\left(t \mid W^{E}, p\right) .
$$

\section{COMPARISON WITH EXISTING BOUNDS}

In Subsection VII-A we compare our exponent $e_{\phi}\left(R \mid W^{E}, p\right)$ with those derived by [17], [6] in the general setting. In Subsections VII-B and VII-C, using discussion in Subsection $\mathrm{V}-\mathrm{B}$, we treat this comparison in special cases more deeply.

\section{A. General case}

Now, we compare the lower obtained bound $e_{\phi}\left(R \mid W^{E}, p\right)$ for the exponential rate of decrease for Eve's distinguishability with existing lower bounds [17], [6]. Using the quantity

$$
\begin{aligned}
\psi(t \mid W, p) & :=\log \sum_{y}\left(\sum_{x} p(x)\left(W_{x}(y)\right)^{1+t}\right) W_{p}(y)^{-t} \\
W_{p}(y) & :=\sum_{x} p(x) W_{x}(y)
\end{aligned}
$$


the previous paper [17] derived the following lower bound of this exponential rate of decrease:

$$
\begin{aligned}
e_{\psi}\left(R \mid W^{E}, p\right) & :=\max _{0 \leq s \leq 1} \frac{s R-\psi\left(s \mid W^{E}, p\right)}{1+s} \\
& =\max _{0 \leq t \leq 1 / 2} t R-(1-t) \psi\left(\frac{t}{1-t} \mid W^{E}, p\right) .
\end{aligned}
$$

The other previous paper [6] also derived the following lower bound:

$$
\max _{0 \leq s \leq 1} s R-\psi\left(s \mid W^{E}, p\right)
$$

for the exponential rate of decrease for the mutual information. By applying a discussion similar to Subsection V-B and Pinsker's inequality (9), the bound (78) yields the bound

$$
\tilde{e}_{\psi}\left(R \mid W^{E}, p\right):=\max _{0 \leq s \leq 1} \frac{s R-\psi\left(s \mid W^{E}, p\right)}{2}
$$

which is smaller than the lower bound $e_{\psi}\left(R \mid W^{E}, p\right)$ because $\frac{1}{2} \leq \frac{1}{1+s}$ for $0 \leq s \leq 1$. Hence, in order to show the superiority of our bound $e_{\phi}\left(R \mid W^{E}, p\right)$, it is sufficient to show the superiority over the bound $e_{\psi}\left(R \mid W^{E}, p\right)$.

In the following, we compare the two bounds $e_{\phi}\left(R \mid W^{E}, p\right)$ and $e_{\psi}\left(R \mid W^{E}, p\right)$. For this purpose, we treat $e^{\phi\left(t \mid W^{E}, p\right)}$ and $e^{(1-t) \psi\left(\frac{t}{1-t} \mid W^{E}, p\right)}$ for $0 \leq t \leq \frac{1}{2}$. The reverse Hölder inequality [28] for the measurable space $(\mathcal{X}, p)$ is

$$
\begin{aligned}
& \sum_{x \in \mathcal{X}} p(x)|X(x) Y(x)| \\
\geq & \left(\sum_{x \in \mathcal{X}} p(x)|X(x)|^{\frac{1}{1+s}}\right)^{1+s}\left(\sum_{x \in \mathcal{X}} p(x)|Y(x)|^{-\frac{1}{s}}\right)^{-s}
\end{aligned}
$$

for $s \geq 0$. Using this inequality, we obtain

$$
\begin{aligned}
& \sum_{y}\left[\sum_{x} p(x)\left(W_{x}(y)\right)^{1+s}\right] W_{p}(y)^{-s} \\
\geq & \left(\sum_{y}\left[\sum_{x} p(x)\left(W_{x}(y)\right)^{1+s}\right]^{\frac{1}{1+s}}\right)^{1+s} \cdot\left(\sum_{y} W_{p}(y)^{-s \cdot-\frac{1}{s}}\right)^{-s} \\
= & \left(\sum_{y}\left[\sum_{x} p(x)\left(W_{x}(y)\right)^{1+s}\right]^{\frac{1}{1+s}}\right)^{1+s} .
\end{aligned}
$$

Substituting $s=\frac{t}{1-t}$, we obtain

$$
\begin{aligned}
& \sum_{y}\left[\sum_{x} p(x)\left(W_{x}(y)\right)^{\frac{1}{1-t}}\right] W_{p}(y)^{\frac{-t}{1-t}} \\
\geq & \left(\sum_{y}\left[\sum_{x} p(x)\left(W_{x}(y)\right)^{\frac{1}{1-t}}\right]^{1-t}\right)^{\frac{1}{1-t}},
\end{aligned}
$$

which implies

$$
\begin{aligned}
& e^{(1-t) \psi\left(\frac{t}{1-t} \mid W^{E}, p\right)} \\
= & \left(\sum_{y}\left[\sum_{x} p(x)\left(W_{x}(y)\right)^{\frac{1}{1-t}}\right] W_{p}(y)^{\frac{-t}{1-t}}\right)^{1-t} \\
\geq & \sum_{y}\left[\sum_{x} p(x)\left(W_{x}(y)\right)^{\frac{1}{1-t}}\right]^{1-t}=e^{\phi\left(t \mid W^{E}, p\right)} .
\end{aligned}
$$

Thus, our bound $e_{\phi}\left(R \mid W^{E}, p\right)$ for the exponential rate of decrease is better than the existing bound $e_{\psi}\left(R \mid W^{E}, p\right)$ [17].

Example 1: Assume that $\mathcal{X}=\mathcal{E}=\{0,1\}$. We consider the following channel.

$W_{0}(0)=\alpha, W_{0}(1)=1-\alpha, W_{1}(0)=1-9 \alpha, W_{1}(1)=9 \alpha$.

When $p(0)=1 / 2, p(1)=1 / 2$,

$$
\begin{aligned}
I(p, W)= & h(1 / 2-5 \alpha)-\frac{(h(\alpha)+h(9 \alpha)}{2} \\
\psi(t \mid p, W)= & \log \left(\left(\frac{\alpha^{1+t}+(1-9 \alpha)^{1+t}}{2}\left(\frac{1}{2}-5 \alpha\right)^{-t}\right.\right. \\
& \left.+\left(\frac{(9 \alpha)^{1+t}+(1-\alpha)^{1+t}}{2}(1 / 2+5 \alpha)^{-t}\right)\right) \\
\phi(t \mid p, W)= & \log \left(\left(\frac{\alpha^{1 /(1-t)}+(1-9 \alpha)^{1 /(1-t)}}{2}\right)^{1-t}\right. \\
& \left.+\left(\frac{(9 \alpha)^{1 /(1-t)}+(1-\alpha)^{1 /(1-t)}}{2}\right)^{1-t}\right) .
\end{aligned}
$$

Then, the three bounds $e_{\phi}(R \mid W, p), \quad e_{\psi}(R \mid W, p)$, and $\tilde{e}_{\psi}(R \mid W, p)$ with $\alpha=0.05$ are numerically compared as in Fig. 4

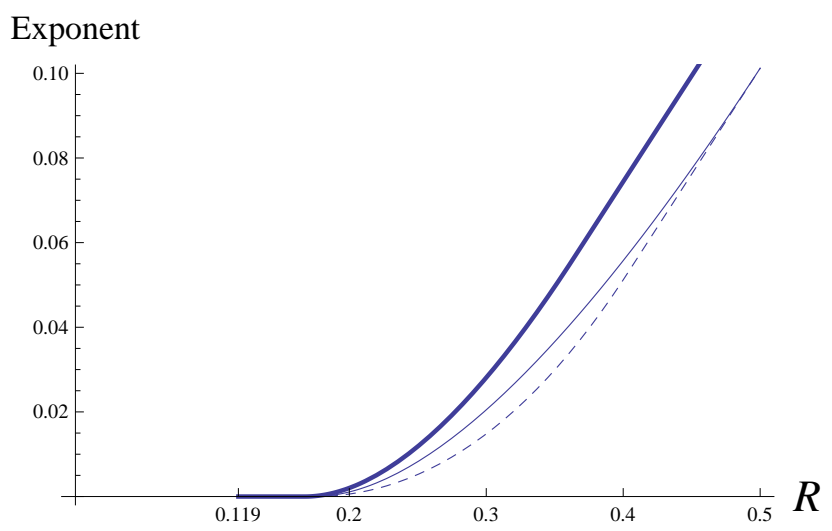

Fig. 4. Lower bounds of exponent. Thick line: $e_{\phi}(R \mid W, p)$ (The present paper), Normal line: $e_{\psi}(R \mid W, p)$ [17], Dashed line: $\tilde{e}_{\psi}(R \mid W, p)$ [6]. Here, $\alpha$ is chosen to be 0.0500 . Then, $I(p, W)=0.119$.

\section{B. Additive case}

Next, we consider a more specific case. When $\mathcal{X}=\mathcal{Z}$ and $\mathcal{X}$ is a module and $W_{x}(z)=W_{0}(z-x)=P^{X}(z-x)$, the channel $W$ is called additive. 
Since

$$
\begin{aligned}
& e^{(1-t) \psi\left(\frac{t}{1-t} \mid W^{E}, p_{\text {mix }}\right)}=e^{\phi\left(t \mid W^{E}, p_{\text {mix }}\right)} \\
& =|\mathcal{X}|^{t} e^{-t H} \frac{1}{1-t}\left(X \mid P^{X}\right),
\end{aligned}
$$

any additive channel $W^{E}$ satisfies

$$
\begin{aligned}
& e_{\psi}\left(R \mid W^{E}, p_{\operatorname{mix}}\right)=e_{\phi}\left(R \mid W^{E}, p_{\operatorname{mix}}\right) \\
= & \left.\max _{0 \leq t \leq \frac{1}{2}} t(R-\log |\mathcal{X}|)+t H_{\frac{1}{1-t}}\left(X \mid P^{X}\right)\right) \\
= & e_{H}\left(X\left|P^{X}\right| \log |\mathcal{X}|-R\right)
\end{aligned}
$$

and

$$
\begin{aligned}
& \tilde{e}_{\psi}\left(R \mid W^{E}, p_{\text {mix }}\right)=\max _{0 \leq t \leq \frac{1}{2}} \frac{t(R-\log |\mathcal{X}|)+t H_{\frac{1}{1-t}}\left(X \mid P^{X}\right)}{2-2 t} \\
= & \tilde{e}_{H}\left(X\left|P^{X}\right| \log |\mathcal{X}|-R\right)
\end{aligned}
$$

for the uniform distribution $p_{\operatorname{mix}}$ on $\mathcal{X}$.

Hence, our bound $e_{\phi}\left(R \mid W^{E}, p_{\text {mix }}\right)$ is the same as the previous bound $e_{\psi}\left(R \mid W^{E}, p_{\text {mix }}\right)$. However, since $\frac{1}{2-2 t}<1$ for $t \in[0,1 / 2)$, our bound $e_{\phi}\left(R \mid W^{E}, p_{\text {mix }}\right)$ is strictly better than the bound $\tilde{e}_{\psi}\left(R \mid W^{E}, p_{\text {mix }}\right)$ by the other previous paper [6] when the maximum is attained by $t \in[0,1 / 2)$.

\section{General additive case}

We consider a more general case. Eve is assumed to have two random variables $Z \in \mathcal{X}$ and $Z^{\prime} \in \mathcal{Z}^{\prime}$. The first random variable $Z$ is the output of an additive channel depending on the second variable $Z^{\prime}$. That is, the channel $W_{x}^{E}\left(z, z^{\prime}\right)$ can be written as $W_{x}^{E}\left(z, z^{\prime}\right)=P^{X, Z^{\prime}}\left(z-x, z^{\prime}\right)$, where $P^{X, Z^{\prime}}$ is a joint distribution. Hereafter, this channel model is called a general additive channel. This channel is also called a regular channel [21]. For this channel model, we obtain

$$
\begin{aligned}
& e^{\phi\left(s \mid W^{E}, P_{\mathrm{mix}}, \mathcal{X}\right)}=\sum_{z, z^{\prime}}\left(\sum_{x} \frac{1}{|\mathcal{X}|} W_{x}^{E}\left(z, z^{\prime}\right)^{\frac{1}{1-s}}\right)^{1-s} \\
= & \sum_{z, z^{\prime}}\left(\sum_{x} \frac{1}{|\mathcal{X}|} P^{X, Z^{\prime}}\left(z-x, z^{\prime}\right)^{\frac{1}{1-s}}\right)^{1-s} \\
= & \frac{1}{|\mathcal{X}|^{1-s}} \sum_{z, z^{\prime}}\left(\sum_{x} P^{X, Z^{\prime}}\left(-x, z^{\prime}\right)^{\frac{1}{1-s}}\right)^{1-s} \\
= & \frac{|\mathcal{X}|}{|\mathcal{X}|^{1-s}} \sum_{z^{\prime}}\left(\sum_{x} P^{X, Z^{\prime}}\left(x, z^{\prime}\right)^{\frac{1}{1-s}}\right)^{1-s} \\
= & \left.|\mathcal{X}|^{s} e^{\phi\left(s|X| Z^{\prime} \mid P^{X, Z^{\prime}}\right.}\right)
\end{aligned}
$$

and

$$
\begin{aligned}
& e^{\psi\left(s \mid W^{E}, p_{\text {mix }}\right)} \\
= & \sum_{z, z^{\prime}}\left(\sum_{x} \frac{1}{|\mathcal{X}|} W_{x}^{E}\left(z, z^{\prime}\right)^{1+s}\right)\left(\sum_{x} \frac{1}{|\mathcal{X}|} W_{x}^{E}\left(z, z^{\prime}\right)\right)^{-s} \\
= & \left.\mathcal{X}\right|^{s-1} \sum_{z, z^{\prime}}\left(\sum_{x} P^{X, Z^{\prime}}\left(z-x, z^{\prime}\right)^{1+s}\right)\left(\sum_{x} P^{X, Z^{\prime}}\left(z-x, z^{\prime}\right)\right)^{-s} \\
= & |\mathcal{X}|^{s-1} \sum_{z, z^{\prime}}\left(\sum_{x} P^{X, Z^{\prime}}\left(-x, z^{\prime}\right)^{1+s}\right) P^{Z^{\prime}}\left(z^{\prime}\right)^{-s} \\
= & |\mathcal{X}|^{s-1}|\mathcal{X}| \sum_{z^{\prime}} \sum_{x} P^{X, Z^{\prime}}\left(x, z^{\prime}\right)^{1+s} P^{Z^{\prime}}\left(z^{\prime}\right)^{-s} \\
= & |\mathcal{X}|^{s} e^{-s H_{1+s}\left(X\left|Z^{\prime}\right| P^{X, Z^{\prime}}\right)}
\end{aligned}
$$

Then, the equalities

$$
\begin{aligned}
& e_{\phi}\left(R \mid W^{E}, p_{\text {mix }}\right) \\
= & \max _{0 \leq t \leq \frac{1}{2}} t(R-\log |\mathcal{X}|)-\phi\left(t|X| Z^{\prime} \mid P^{X, Z^{\prime}}\right) \\
= & e_{\phi}\left(X\left|Z^{\prime}\right| P^{X, Z^{\prime}}|\log | \mathcal{X} \mid-R\right), \\
& e_{\psi}\left(R \mid W^{E}, p_{\text {mix }}\right) \\
= & \max _{0 \leq t \leq \frac{1}{2}} t(R-\log |\mathcal{X}|)+t H_{\frac{1}{1-t}}\left(X\left|Z^{\prime}\right| P^{X, Z^{\prime}}\right) \\
= & e_{H}\left(X\left|Z^{\prime}\right| P^{X, Z^{\prime}}|\log | \mathcal{X} \mid-R\right), \\
& \tilde{e}_{\psi}\left(R \mid W^{E}, p_{\text {mix }}\right) \\
= & \max _{0 \leq t \leq \frac{1}{2}} \frac{t(R-\log |\mathcal{X}|)+t H_{\frac{1}{1-t}}\left(X\left|Z^{\prime}\right| P^{X, Z^{\prime}}\right)}{2-2 t} \\
= & \tilde{e}_{H}\left(X\left|Z^{\prime}\right| P^{X, Z^{\prime}}|\log | \mathcal{X} \mid-R\right)
\end{aligned}
$$

hold.

Hence, the observation in Section $\mathrm{V}-\mathrm{B}$ can be applied to the comparison among $e_{\phi}\left(R \mid W^{E}, p_{\text {mix }}\right), e_{\psi}\left(R \mid W^{E}, p_{\text {mix }}\right)$, and $\tilde{e}_{\psi}\left(R \mid W^{E}, p_{\text {mix }}\right)$. Due to Lemma $8, e_{\phi}\left(R \mid W^{E}, p_{\text {mix }}\right)$ is strictly better than $e_{\psi}\left(R \mid W^{E}, p_{\text {mix }}\right)$ and $\tilde{e}_{\psi}\left(R \mid W^{E}, p_{\text {mix }}\right)$ except for the special case mentioned in Lemma 8.

\section{WIRE-TAP CHANNEL WITH LINEAR CODING}

In a practical sense, we need to take into account the decoding time. For this purpose, we often restrict our codes to linear codes. In the following, we consider the case where the sender's space $\mathcal{X}$ has the structure of a module. When an error correcting code is given as a submodule $C_{1} \subset \mathcal{X}$ and the decoder by the authorized receiver is given as $\left\{\mathcal{D}_{x}\right\}_{x \in C_{1}}$, our code for a wire-tap channel is given as $\Phi_{C_{1}, C_{2}}=\left(\left|C_{1} / C_{2}\right|,\left\{Q_{[x]}\right\}_{[x] \in C_{1} / C_{2}},\left\{\mathcal{D}_{[x]}\right\}_{[x] \in C_{1} / C_{2}}\right)$ based on a submodule $C_{2}$ of $C_{1}$ as follows. The encoding $Q_{[x]}$ is given as the uniform distribution on the coset $[x]:=x+C_{2}$, and the decoding $\mathcal{D}_{[x]}$ is given as the subset $\cup_{x^{\prime} \in x+C_{2}} \mathcal{D}_{x^{\prime}}$. Next, we consider a submodule $C_{2}(\mathbf{X})$ of $C_{1}$ with cardinality $\left|C_{2}(\mathbf{X})\right|=L$ that is labeled by a random variable $\mathbf{X}$. Then, the module $C_{2}(\mathbf{X})$ can be regarded as a random variable. Now, we impose the module $C_{2}(\mathbf{X})$ the following condition.

Condition 4: Any element $x \neq 0 \in C_{1}$ is included in $C_{2}(\mathbf{X})$ with probability at most $\frac{L}{\mid C_{1}}$.

Then, using (75), we can evaluate the performance of the constructed code in the following way.

Theorem 6: Choose the subcode $C_{2}(\mathbf{X})$ according to Condition 4 We construct the code $\Phi_{C_{1}, C_{2}}(\mathbf{X})$ by choosing the distribution $Q_{[x]}$ to be the uniform distribution on $[x]$ for $[x] \in C_{1} / C_{2}(\mathbf{X})$. Then, we obtain

$$
\mathrm{E}_{\mathbf{X}} d_{1}\left(\Phi_{C_{1}, C_{2}(\mathbf{X})} \mid E\right) \leq 3 \frac{e^{\phi\left(t \mid W^{E}, P_{\text {mix }, C_{1}}\right)}}{L^{t}} \quad 0 \leq \forall t \leq \frac{1}{2}
$$

where $P_{\text {mix }, S}$ is the uniform distribution on the subset $S$.

When the channel $W^{E}$ is additive, i.e., $W_{x}^{E}(z)=P^{X}(z-$ $x)$, the equation $\phi\left(t \mid W^{E}, P_{\operatorname{mix}, C_{1}+x}\right)=\phi\left(t \mid W^{E}, P_{\operatorname{mix}, C_{1}}\right)$ holds for any $x$. Thus, the concavity of $\left.e^{\phi\left(t \mid W^{E}\right.}, p\right)$ (Lemma 9) implies that

$$
\phi\left(t \mid W^{E}, P_{\operatorname{mix}, C_{1}}\right) \leq \phi\left(t \mid W^{E}, P_{\operatorname{mix}, \mathcal{X}} .\right.
$$


Thus, combining (87), (88), and (80), we obtain

$$
\mathrm{E}_{\mathbf{X}} d_{1}\left(\Phi_{C_{1}, C_{2}(\mathbf{X})} \mid E\right) \leq 3 \frac{|\mathcal{X}|^{t} e^{-t H} \frac{1}{1-t}(X \mid P)}{L^{t}}
$$

for $0<t<\frac{1}{2}$. That is, when $L=e^{R}$, taking the minimum concerning $0<t<\frac{1}{2}$, we obtain

$$
\mathrm{E}_{\mathbf{X}} d_{1}\left(\Phi_{C_{1}, C_{2}(\mathbf{X})} \mid E\right) \leq 3 e^{-e_{H}\left(X\left|P^{X}\right| \log |\mathcal{X}|-R\right)} .
$$

When the additive noise obeys the $n$-fold i.i.d. of $P$ on $\mathcal{X}^{n}$ and $L=e^{n R}$, we obtain

$$
\mathrm{E}_{\mathbf{X}} d_{1}\left(\Phi_{C_{1}, C_{2}(\mathbf{X})} \mid E\right) \leq 3 e^{-n e_{H}\left(X\left|P^{X}\right| \log |\mathcal{X}|-R\right)} .
$$

Similarly, when the channel $W^{E}$ is general additive, i.e., $W_{x}^{E}\left(z, z^{\prime}\right)=P^{X, Z^{\prime}}\left(z-x, z^{\prime}\right)$, combining (87), (88), and (82), we obtain

$$
\mathrm{E}_{\mathbf{X}} d_{1}\left(\Phi_{C_{1}, C_{2}(\mathbf{X})} \mid E\right) \leq 3 \frac{|\mathcal{X}|^{t} e^{\phi\left(t|X| Z^{\prime} \mid P^{X, Z^{\prime}}\right)}}{L^{t}}
$$

for $0<t<\frac{1}{2}$. That is, when $L=e^{R}$, taking the minimum concerning $0<t<\frac{1}{2}$, we obtain

$$
\mathrm{E}_{\mathbf{X}} d_{1}\left(\Phi_{C_{1}, C_{2}(\mathbf{X})} \mid E\right) \leq 3 e^{-e_{\phi}\left(X\left|Z^{\prime}\right| P^{X, Z^{\prime}}|\log | \mathcal{X} \mid-R\right)} .
$$

In the $n$-fold i.i.d. case, when $L=e^{n R}$, we obtain

$$
\mathrm{E}_{\mathbf{X}} d_{1}\left(\Phi_{C_{1}, C_{2}(\mathbf{X})} \mid E\right) \leq 3 e^{-n e_{\phi}\left(X\left|Z^{\prime}\right| P^{X, Z^{\prime}}|\log | \mathcal{X} \mid-R\right)} .
$$

When $\mathcal{X}$ is an $n$-dimensional vector space $\mathbb{F}_{q}^{n}$ over the finite field $\mathbb{F}_{q}$, the bound can be attained by the combination of linear code and the concatenation of a Toeplitz matrix and the identity $(\mathbf{X}, I)$ of the size $m \times(m-k)$ [6]. Hence, if the error correcting code $C_{1}$ can be realizable, the whole process in the above code can be realizable.

Remark 1: In the additive case, due to 81 , the exponent of the upper bound given in 91 is the same as that given by the previous paper [17]. However, the code given in [17] is constructed by completely random coding. However, the code given in this section is based on the ordinary linear code. For security, it requires only the universal hash condition. So, our construction requires smaller complexity than that given in [17]. In the general additive case, our exponents (94) is strictly better than that given in [17], which is calculated in (85).

Next, we consider the relation with the other previous paper [6] in the general additive case. The protocol given in [6] is is quite similar to ours. However, as is shown in Lemma 8, except for the very special case, our exponent (94) is strictly better than that given in [6], which is calculated in (86). Remember that the exponent given in [6] is $\tilde{e}_{\psi}\left(R \mid W^{E}, p_{\text {mix }}\right)$, which is mentioned around (79).

\section{SECRET KEY GENERATION With PUbliC COMMUNICATION}

Furthermore, the above result can be applied to secret key generation (distillation) with one-way public communication, in which, Alice, Bob, and Eve are assumed to have initial random variables $A \in \mathcal{A}, B \in \mathcal{B}$, and $E \in \mathcal{E}$, respectively. The task for Alice and Bob is to share a common random variable almost independent of Eve's random variable $E$ by using a public communication. For this purpose, we assume that Alice and Bob can perform local data processing in the both sides and Alice can send messages to Bob via public channel. That is, only one-way communication is allowed. We call such a combination of these operations a code and denote it by $\Phi$.

The quality is evaluated by three quantities: the size of the final common random variable, the probability that their final variables coincide, and Eve's distinguishability $d_{1}(\Phi \mid E)$ of the final joint distribution between Alice and Eve.

In order to construct a protocol for this task, we assume that the set $\mathcal{A}$ has a module structure (any finite set can be regarded as a cyclic group). Then, the objective of secret key distillation can be realized by applying the code of a wire-tap channel as follows. First, Alice generates another uniform random variable $X$ and sends the random variable $X^{\prime}:=X+A$. Then, the distribution of the random variables $B, X^{\prime}\left(E, X^{\prime}\right)$ accessible to Bob (Eve) can be regarded as the output distribution of the channel $x \mapsto W_{x}^{B}\left(x \mapsto W_{x}^{E}\right)$. The channels $W^{B}$ and $W^{E}$ are given as follows.

$W_{x}^{B}\left(x^{\prime}, b\right)=P^{A, B}\left(x^{\prime}-x, b\right), W_{x}^{E}\left(x^{\prime}, e\right)=P^{A, E}\left(x^{\prime}-x, e\right)$,

where $P^{A B}(a, b)\left(P^{A E}(a, e)\right)$ is the joint probability between Alice's initial random variable $A$ and Bob's (Eve's) initial random variable $B(E)$. Hence, the channel $W^{E}$ is general additive.

Applying Theorem 5 to the uniform distribution $P_{\mathrm{mix}}^{A}$, for any numbers $M$ and $L$, due to $(82)$, there exists a code $\Phi$ such that $|\Phi|=M$ and 1

$$
\begin{aligned}
\epsilon_{B}(\Phi) & \leq 3 \min _{0 \leq s \leq 1}(M L)^{s}|\mathcal{A}|^{-s} e^{\phi\left(-s|A| B \mid P^{A, B}\right)} \\
d_{1}(\Phi \mid E) & \leq 9 \min _{0 \leq t \leq \frac{1}{2}} \frac{|\mathcal{A}|^{t} e^{\phi\left(t|A| E \mid P^{A, E}\right)}}{L^{t}}
\end{aligned}
$$

In particular, when the joint distribution between $A$ and $B(E)$ is the $n$-fold independent and identical distribution (i.i.d.) of $P^{A, B}\left(P^{A, E}\right)$, respectively, the relation $\phi\left(t\left|A^{n}\right| E^{n} \mid\left(P^{A, E}\right)^{n}\right)=n \phi\left(t|A| E \mid P^{A, E}\right)$ hold. Thus, there exists a code $\Phi_{n}$ for any integers $L_{n}, M_{n}$, and any probability distribution $p$ on $\mathcal{X}$ such that $\left|\Phi_{n}\right|=M_{n}$ and

$$
\begin{aligned}
\epsilon_{B}(\Phi) & \leq 3 \min _{0 \leq s \leq 1}\left(M_{n} L_{n}\right)^{s}|\mathcal{A}|^{-n s} e^{n \phi\left(-s|A| B \mid P^{A, B}\right)} \\
d_{1}\left(\Phi_{n} \mid E\right) & \leq 9 \min _{0 \leq t \leq \frac{1}{2}} \frac{\left.|\mathcal{A}|^{n t} e^{n \phi\left(t|A| E \mid P^{A, E}\right.}\right)}{L_{n}^{t}}
\end{aligned}
$$

Finally, we mention the relation with the previous paper [17]. Since the above discussion is an application of section VIII, the same comparison as Remark 1] is valid. Hence, our evaluation (99) is strictly better than that given in [17] except for the special case.

1 The previous paper [17 Section VI] derived upper bounds different from 96 and 98, while it treat the same protocol. The previous paper [17 Section VI] erroneously calculated $e^{\phi\left(-s \mid W^{B}, P_{\text {mix }, \mathcal{A}}\right)}$ to $|\mathcal{A}|^{-s} e^{-s H} \frac{1}{1+s}\left(A|B| P^{A, B}\right)$. However, the correct calculation is $|\mathcal{A}|^{-s} e^{\phi\left(-s|A| B \mid P^{A, B}\right)}$ as is shown in 82 . 


\section{DisCUSSION}

We have derived a tight evaluation of the exponent for the average of the $L_{1}$ norm distance between the generated random number and the uniform random number when universal ${ }_{2}$ hash functions are applied and the key generation rate is less than the critical rate $R_{1}$. Using this evaluation, we have obtained an upper bound for Eve's distinguishability in secret key generation from a common random number without communication when universal ${ }_{2}$ hash functions are applied. Since our bound is based on the Rényi entropy of order $1+s$ for $s \in[0,1]$, it can be regarded as an extension of Bennett et al [2]'s result with the Rényi entropy of order 2 .

Applying this bound to the wire-tap channel, we obtain an upper bound for Eve's distinguishability, which yields an exponential upper bound. This exponent improves on the existing exponent [17]. Further, when the error correction code is given by a linear code and when the channel is additive or general additive, the privacy amplification is given by a concatenation of a Toeplitz matrix and the identity matrix. This method can be applied to secret key distillation with public communication.

\section{ACKNOWLEDGMENTS}

The author is grateful to Professors Ryutaroh Matsumoto and Takeshi Koshiba for a helpful comments. He is also grateful to the referee of the previous version for helpful comments. This research was partially supported by a MEXT Grant-inAid for Young Scientists (A) No. 20686026 and a MEXT Grant-in-Aid for Scientific Research (A) No. 23246071. The Centre for Quantum Technologies is funded by the Singapore Ministry of Education and the National Research Foundation as part of the Research Centres of Excellence programme.

\section{APPENDIX A}

PROOF OF THEOREM 2

First, for a fixed element $a \in \Omega$, we introduce the condition for a hash function $f_{\mathbf{X}}$ :

Condition 5 (Condition $[a, \Omega]$ ):

$$
f_{\mathbf{X}}(a) \neq f_{\mathbf{X}}\left(a^{\prime}\right) \text { for } \forall a^{\prime}(\neq a) \in \Omega .
$$

Let $P[a, \Omega]$ be the probability that Condition $[a, \Omega]$ holds. Due to the strongly universal ${ }_{2}$ condition, it is evaluated as

$$
\begin{aligned}
1-P[a, \Omega] & =\operatorname{Pr} \cup_{a^{\prime}(\neq a) \in \Omega}\left\{f_{\mathbf{X}}(a)=f_{\mathbf{X}}\left(a^{\prime}\right)\right\} \\
& \leq \sum_{a^{\prime}(\neq a) \in \Omega} \operatorname{Pr}\left\{f_{\mathbf{X}}(a)=f_{\mathbf{X}}\left(a^{\prime}\right)\right\} \\
& =\sum_{a^{\prime}(\neq a) \in \Omega} \frac{1}{M}=\frac{|\Omega|}{M},
\end{aligned}
$$

which implies that $P[a, \Omega] \geq 1-\frac{|\Omega|}{M}$. When we denote the expectation concerning the hash funcations under Condition $[a, \Omega]$ by $\mathrm{E}_{\mathbf{X} \mid[a, \Omega]}$, the strongly universal ${ }_{2}$ condition yields that

$$
\begin{aligned}
& \mathrm{E}_{\mathbf{X} \mid[a, \Omega]}\left(P^{A}(a)+\sum_{a^{\prime}(\neq a) \in f_{\mathbf{X}}^{-1}(a)} P^{A}\left(a^{\prime}\right)-\frac{1}{M}\right) \\
= & P^{A}(a)+\mathrm{E}_{\mathbf{X} \mid[a, \Omega]} \sum_{a^{\prime}(\neq a) \in f_{\mathbf{X}}^{-1}(a)} P^{A}\left(a^{\prime}\right)-\frac{1}{M} \\
= & P^{A}(a)+\frac{1}{M} \sum_{a^{\prime}(\neq a) \in \mathcal{A} \backslash \Omega} P^{A}\left(a^{\prime}\right)-\frac{1}{M} \\
= & P^{A}(a)+\frac{1}{M}\left(1-P^{A}(\Omega)\right)-\frac{1}{M} \\
= & P^{A}(a)-\frac{1}{M} P^{A}(\Omega) .
\end{aligned}
$$

When Conditions $\left[a_{1}, \Omega\right],\left[a_{2}, \Omega\right], \ldots,\left[a_{k}, \Omega\right]$ hold for $a_{1}, a_{2}, \ldots, a_{k} \in \Omega, f_{\mathbf{X}}\left(a_{1}\right), f_{\mathbf{X}}\left(a_{2}\right), \ldots, f_{\mathbf{X}}\left(a_{k}\right)$ are different. Then,

$$
d_{1}\left(P^{f_{\mathbf{X}}(A)}\right) \geq \sum_{j=1}^{k}\left|P^{A}\left(a_{j}\right)+\sum_{a^{\prime}\left(\neq a_{j}\right) \in f_{\mathbf{X}}^{-1}\left(a_{j}\right)} P^{A}\left(a^{\prime}\right)-\frac{1}{M}\right| .
$$

Now, we define the random variable $Y(a)$ to be 1 when Condition $[a, \Omega]$ holds. We define it to be 0 otherwise. Then,

$$
\begin{aligned}
& d_{1}\left(P^{f_{\mathbf{X}}(A)}\right) \\
\geq & \sum_{a \in \Omega} Y(a)\left|P^{A}(a)+\sum_{a^{\prime}(\neq a) \in f_{\mathbf{X}}^{-1}(a)} P^{A}\left(a^{\prime}\right)-\frac{1}{M}\right| .
\end{aligned}
$$

Therefore, taking the expectation, we can evaluate $\mathrm{E}_{\mathbf{X}} d_{1}\left(P^{f \mathbf{X}(A)}\right)$ as follows.

$$
\begin{aligned}
& \mathrm{E}_{\mathbf{X}} d_{1}\left(P^{f_{\mathbf{X}}(A)}\right) \\
\geq & \sum_{a \in \Omega} P[a, \Omega] \mathrm{E}_{\mathbf{X} \mid[a, \Omega]}\left|P^{A}(a)+\sum_{a^{\prime}(\neq a) \in f_{\mathbf{X}}^{-1}(a)} P^{A}\left(a^{\prime}\right)-\frac{1}{M}\right| \\
\geq & \sum_{a \in \Omega} P[a, \Omega] \mathrm{E}_{\mathbf{X} \mid[a, \Omega]}\left(P^{A}(a)+\sum_{a^{\prime}(\neq a) \in f_{\mathbf{X}}^{-1}(a)} P^{A}\left(a^{\prime}\right)-\frac{1}{M}\right) \\
= & \sum_{a \in \Omega}\left(1-\frac{|\Omega|}{M}\right)\left(P^{A}(a)-\frac{1}{M} P^{A}(\Omega)\right) \\
= & \left(1-\frac{|\Omega|}{M}\right)\left(P^{A}(\Omega)-\frac{|\Omega|}{M} P^{A}(\Omega)\right)=\left(1-\frac{|\Omega|}{M}\right)^{2} P^{A}(\Omega) .
\end{aligned}
$$

\section{APPENDIX B}

ProOF OF LEMMA 6

We choose $s(R)$ such that $\left.\frac{d\left(s H_{1+s}(A \mid P)\right)}{d s}\right|_{s=s(R)}=$ $H\left(P_{1+s(R)}\right)+D\left(P_{1+s(R)} \| P\right)=R$, where $P_{1+s}(a):=$ 
$\frac{P(a)^{1+s}}{\sum_{a^{\prime}} P\left(a^{\prime}\right)^{1+s}}$. When $Q$ satisfies $H(Q)+D(Q \| P)=R$,

$$
\begin{aligned}
& D(Q \| P)-D\left(P_{1+s} \| P\right) \\
= & \sum_{a} Q(a)(\log Q(a)-\log P(a)) \\
& -\sum_{a} \frac{P(a)^{1+s}}{\sum_{a^{\prime}} P\left(a^{\prime}\right)^{1+s}}\left(\log \frac{P(a)^{1+s}}{\sum_{a^{\prime}} P\left(a^{\prime}\right)^{1+s}}-\log P(a)\right) \\
= & \sum_{a} Q(a)\left(\log Q(a)-\log \frac{P(a)^{1+s}}{\sum_{a^{\prime}} P\left(a^{\prime}\right)^{1+s}}\right) \\
& +\sum_{a}\left(Q(a)-\frac{P(a)^{1+s}}{\sum_{a^{\prime}} P\left(a^{\prime}\right)^{1+s}}\right) \\
& \cdot\left(\log \frac{P(a)^{1+s}}{\sum_{a^{\prime}} P\left(a^{\prime}\right)^{1+s}}-\log P(a)\right) \\
= & D\left(Q \| P_{1+s}\right)+s \sum_{a}\left(Q(a)-\frac{P(a)^{1+s}}{\sum_{a^{\prime}} P\left(a^{\prime}\right)^{1+s}}\right) \log P(a) \\
= & D\left(Q \| P_{1+s}\right) \\
& +s\left(H\left(P_{1+s}\right)+D\left(P_{1+s} \| P\right)-H(Q)+D(Q \| P)\right) \\
= & D\left(Q \| P_{1+s}\right) \geq 0 .
\end{aligned}
$$

Hence,

$$
\begin{aligned}
& \min _{Q: H(Q)+D(Q \| P)=R} H(Q)+2 D(Q \| P)-R \\
= & \min _{Q: H(Q)+D(Q \| P)=R} D(Q \| P)=D\left(P_{1+s(R)} \| P\right) \\
= & s H_{1+s}(A \mid P)-\left.s(R) \frac{d\left(s H_{1+s}(A \mid P)\right)}{d s}\right|_{s=s(R)} \\
= & s H_{1+s}(A \mid P)-s(R) R=\max _{0 \leq s} s H_{1+s}(A \mid P)-s R .
\end{aligned}
$$

The last equation follows from the concavity of $s H_{1+s}(A \mid P)$ concerning $s$.

Assume that $\left.\frac{d\left(s H_{1+s}(A \mid P)\right)}{d s}\right|_{s=1} \leq R$. Then, $s(R) \leq 1$. When $R^{\prime} \geq R$,

$$
\begin{aligned}
& \min _{Q: H(Q)+D(Q \| P)=R^{\prime}} H(Q)+2 D(Q \| P)-R \\
= & \max _{0 \leq s} s H_{1+s}(A \mid P)-s R+R^{\prime}-R \\
\geq & s H_{1+s(R)}(A \mid P)-s(R) R^{\prime}+R^{\prime}-R \\
\geq & s H_{1+s(R)}(A \mid P)-s(R) R \\
= & \max _{0 \leq s} s H_{1+s}(A \mid P)-s R \\
= & \max _{0 \leq s \leq 1} s H_{1+s}(A \mid P)-s R,
\end{aligned}
$$

which implies (59).

Assume that $\left.\frac{d\left(s H_{1+s}(A \mid P)\right)}{d s}\right|_{s=1}>R$. When $R^{\prime} \geq R$,

$$
\begin{aligned}
& \min _{Q: H(Q)+D(Q \| P)=R^{\prime}} H(Q)+2 D(Q \| P)-R \\
= & \max _{0 \leq s} s H_{1+s}(A \mid P)-s R+R^{\prime}-R \\
\geq & 1 H_{1+1}(A \mid P)-R^{\prime}+R^{\prime}-R=H_{2}(A \mid P)-R .
\end{aligned}
$$

Further, when $R^{\prime}=\left.\frac{d\left(s H_{1+s}(A \mid P)\right)}{d s}\right|_{s=1}$,

$$
\begin{aligned}
& \min _{Q: H(Q)+D(Q \| P)=R^{\prime}} H(Q)+2 D(Q \| P)-R \\
= & H_{1+1}(A \mid P)-R^{\prime}+R^{\prime}-R=H_{2}(A \mid P)-R,
\end{aligned}
$$

which implies (60).
Further, the concavity of $s \mapsto s H_{1+s}(A \mid P)$ and the condition $\left.\frac{d\left(s H_{1+s}(A \mid P)\right)}{d s}\right|_{s=1}>R$ imply that $\max _{0 \leq s \leq 1} s H_{1+s}(A \mid P)-s R=H_{2}(A \mid P)-R$. Thus, we obtain 61.

\section{APPENDiX C \\ PROOF OF 37}

First, we consider the the minimum $\min _{\tilde{P}^{A}: H_{\min }\left(A \mid \tilde{P}^{A}\right) \geq R^{\prime}} d_{1}\left(P^{A}, \tilde{P}^{A}\right)$, where $\tilde{P}^{A}$ is chosen to be a subdistribution satisfying $H_{\min }\left(A \mid \tilde{P}^{A}\right) \geq R^{\prime}$.

$$
\begin{aligned}
& \min _{\tilde{P}^{A}: H_{\min }\left(A \mid \tilde{P}^{A}\right) \geq R^{\prime}} d_{1}\left(P^{A}, \tilde{P}^{A}\right) \\
= & \min _{\tilde{P}^{A}: H_{\min }\left(A \mid \tilde{P}^{A}\right) \geq R^{\prime}} \sum_{a \in \mathcal{A}}\left|P^{A}(a)-\tilde{P}^{A}(a)\right| \\
= & \sum_{a \in \mathcal{A}: P^{A}(a)>e^{-R^{\prime}}}\left(P^{A}(a)-e^{-R}\right) \\
\geq & \frac{1}{2} \sum_{a \in \mathcal{A}: P^{A}(a)>2 e^{-R^{\prime}}} P^{A}(a)=\frac{1}{2} P^{A}\left\{P^{A}(a)>2 e^{-R^{\prime}}\right\} .
\end{aligned}
$$

Using this relation, we have

$$
\begin{aligned}
& \min _{\epsilon}\left(M^{\frac{1}{2}} e^{-\frac{H_{\min , \epsilon}\left(A \mid\left(P^{A}\right)\right.}{2}}+2 \epsilon\right) \\
= & \min _{R^{\prime}} \tilde{P}_{\tilde{P}^{A}: H_{\min }\left(A \mid \tilde{P}^{A}\right) \geq R^{\prime}}\left(M^{\frac{1}{2}} e^{-R^{\prime} / 2}+2 d_{1}\left(P^{A}, \tilde{P}^{A}\right)\right) \\
= & \min _{R^{\prime}}\left(M^{\frac{1}{2}} e^{-R^{\prime} / 2}+P^{A}\left\{P^{A}(a)>2 e^{-R^{\prime}}\right\}\right)
\end{aligned}
$$

Using (29), we obtain

$$
\begin{aligned}
& \lim _{n \rightarrow \infty} \frac{-1}{n} \log \min _{R^{\prime}}\left(e^{\frac{n R-n R^{\prime}}{2}}+\left(P^{A}\right)^{2}\left\{\left(P^{A}\right)^{n}(a)>2 e^{-n R^{\prime}}\right\}\right) \\
= & \max _{R^{\prime}} \lim _{n \rightarrow \infty} \frac{-1}{n} \log \left(e^{\frac{n R-n R^{\prime}}{2}}+\left(P^{A}\right)^{2}\left\{\left(P^{A}\right)^{n}(a)>2 e^{-n R^{\prime}}\right\}\right) \\
= & \max _{R^{\prime}} \min \left(R^{\prime}-R, \max _{0 \leq s} s H_{1+s}\left(A \mid P^{A}\right)-s R^{\prime}\right) \\
= & \max _{R^{\prime}} \max _{0 \leq s} \min \left(R^{\prime}-R, s H_{1+s}\left(A \mid P^{A}\right)-s R^{\prime}\right) \\
= & \max _{0 \leq s} \max _{R^{\prime}} \min \left(R^{\prime}-R, s H_{1+s}\left(A \mid P^{A}\right)-s R^{\prime}\right) .
\end{aligned}
$$

Since $s H_{1+s}\left(A \mid P^{A}\right)-s R^{\prime}$ is monotonically decreasing with $R^{\prime}$ and $R^{\prime}-R$ is monotonically increasing with $R^{\prime}$, the maximum $\max _{R^{\prime}} \min \left(R^{\prime}-R, s H_{1+s}\left(A \mid P^{A}\right)-s R^{\prime}\right)$ is realized when $R^{\prime}-R=s H_{1+s}\left(A \mid P^{A}\right)-s R^{\prime}$, which implies that $R^{\prime}=\frac{R+2 s H_{1+s}\left(A \mid P^{A}\right)}{1+2 s}$. Hence,

$$
\begin{aligned}
& \max _{0 \leq s} \max _{R^{\prime}} \min \left(R^{\prime}-R, s H_{1+s}\left(A \mid P^{A}\right)-s R^{\prime}\right) \\
= & \max _{0 \leq s} \frac{s H_{1+s}\left(A \mid P^{A}\right)-s R}{1+2 s} .
\end{aligned}
$$

Therefore, combining (100), (101), and (102), we obtain (37) because $\max _{0 \leq s} \frac{s H_{1+s}\left(A \mid P^{A}\right)-s R}{1+2 s}=$ $\max _{0 \leq t \leq 1} \frac{t H_{1 /(1-t)}\left(A \mid P^{A}\right)-t R}{1+t}$.

\section{REFERENCES}

[1] L. Carter and M. Wegman, "Universal classes of hash functions," $J$. Comput. Sys. Sci., 18(2), 143-154, 1979.

[2] C. H. Bennett, G. Brassard, C. Crepeau, and U.M. Maurer, "Generalized privacy amplification," IEEE Trans. Inform. Theory, 41, 1915-1923, 1995. 
[3] U. Maurer and S. Wolf, "Infromation-theoretic key agreement: From weak to strong secrecy for free," Advances in Cryptology-EUROCRYPT 2000, Lecture Notes in Computer Science, vol.1807, pp.351-368, SpringerVerlag (2000).

[4] R. Renner and S. Wolf, "Simple and Tight Bounds for Information Reconciliation and Privacy Amplification," ASIACRYPT 2005, Lecture Notes in Computer Science, Springer-Verlag, vol. 3788, pp. 199-216, 2005.

[5] R. Renner, "Security of Quantum Key Distribution," PhD thesis, Dipl. Phys. ETH, Switzerland, 2005. arXiv:quantph/0512258.

[6] M. Hayashi, "Exponential decreasing rate of leaked information in universal random privacy amplification," IEEE Trans. Inform. Theory, 57(6), 3989-4001, 2011.

[7] R. Ahlswede and I. Csiszár, "Common randomness in information theory and cryptography part 1: Secret sharing," IEEE Trans. Inform. Theory, 39(4), 1121-1132, 1993.

[8] U. Maurer, "Secret key agreement by public discussion from common information," IEEE Trans. Inform. Theory, 39, 733-742, 1993.

[9] J. Muramatsu, "Secret key agreement from correlated source outputs using low density parity check matrices," IEICE Trans. Fundamentals, E89A(7): 2036-2046, 2006.

[10] J. Muramatsu, S. Miyake, "Construction of Codes for Wiretap Channel and Secret Key Agreement from Correlated Source Outputs by Using Sparse Matrices," IEEE Trans. Inform. Theory, 58(2), 671 - 692, 2012.

[11] S. Watanabe, T. Saitou, R. Matsumoto, T. Uyematsu "Strongly Secure Privacy Amplification Cannot Be Obtained by Encoder of SlepianWolf Code," Proceedings of the 2009 IEEE International Symposium on Information Theory, Volume 2, Seoul, Korea, pp. 1298-1302 (2009) (arXiv:0906.2582)

[12] A. D. Wyner, "The wire-tap channel," Bell. Sys. Tech. Jour, 54, 1355$1387,1975$.

[13] I. Csiszár and J. Körner, "Broadcast channels with confidential messages," IEEE Trans. Inform. Theory, 24(3) 339-348, 1979.

[14] I. Csiszár, "Almost Independence and Secrecy Capacity," Problems of Information Transmission, vol.32(1), pp.40-47, 1996.

[15] I. Devetak, "The private classical information capacity and quantum information capacity of a quantum channel," IEEE Trans. Inform. Theory, 51(1), 44-55, 2005.

[16] A. Winter, A. C. A. Nascimento, and H. Imai, "Commitment Capacity of Discrete Memoryless Channels," Proc. 9th Cirencester Crypto and Coding Conf., LNCS 2989, pp 35-51, Springer, Berlin 2003; cs.CR/0304014 (2003)

[17] M. Hayashi, "General non-asymptotic and asymptotic formulas in channel resolvability and identification capacity and its application to wire-tap channel," IEEE Trans. Inform. Theory, 52(4), 1562-1575, 2006.

[18] H. Krawczyk, "LFSR-based hashing and authentication," Advances in Cryptology - CRYPTO '94., Lecture Notes in Computer Science, vol. 839, Springer-Verlag, pp 129-139, 1994.

[19] I. Csiszár and J. Körner, Information theory: Coding Theorem for Discrete Memoryless systems, Academic Press, New York, (1981)

[20] R. G. Gallager, Information Theory and Reliable Communication, John Wiley \& Sons, 1968.

[21] P. Delsarte and P. Piret, "Algebraic constructions of Shannon codes for regular channels," IEEE Trans. Inform. Theory, 28(4), 593-599, 1982.

[22] R. Cannetti, "Universal composable security: a new paradigm for cryptographic protocols," Proc. 42nd IEEE FOCS, pp. 136-145, Oct. 2001.

[23] S. Watanabe, private communication, 2007. (This communication is written in [6. Appendix III])

[24] M. Hayashi, "Exponents of quantum fixed-length pure state source coding," Physical Review A, Vol.66, 032321 (2002).

[25] M. Hayashi, "Second-Order Asymptotics in Fixed-Length Source Coding and Intrinsic Randomness," IEEE Trans. Inform. Theory, 54, 4619 4637, 2008.

[26] S. Vembu and S. Verdú, "Generating random bits from an arbitrary source: fundamental limits," IEEE Trans. Inform. Theory, 41, 1322-1332 (1995).

[27] A. Dembo and O. Zeitouni, Large Deviations Techniques and Applications, (Springer, 1997).

[28] L.P. Kuptsov, "Holder inequality", in Hazewinkel, Michiel, Encyclopaedia of Mathematics, Springer, (2001).

[29] T. Holenstein and R. Renner, "On the randomness of independent experiments," IEEE Trans. Inform. Theory, 57(4), 1865 - 1871, 2011.

[30] J. Håstad, R. Impagliazzo, L. A. Levin, and M. Luby, "A Pseudorandom Generator from any One-way Function,” SIAM J. Comput. 28, 1364 (1999)

[31] T.S. Han, "The reliability functions of the general source with fixedlength coding," IEEE Trans. Inform. Theory, 46, 2117-2132, (2000).
[32] T. S. Han: Information-Spectrum Methods in Information Theory, (Springer-Verlag, New York, 2002) (Originally written in Japanese in 1998).

[33] M. R. Bloch, and J. N. Laneman, "Secrecy from Resolvability," submitted to IEEE Trans. Inform. Theory, arXiv:1105.5419 (2011).

[34] T.-H. Chou, V. Y. F. Tan, and S. C. Draper, "The Sender-Excited Secret Key Agreement Model: Capacity and Error Exponents," submitted to IEEE Trans. Inform. Theory, arXiv:1107.4148 (2011).

[35] C. E. Shannon, R. G. Gallager, and E. R. Berlekamp, "Lower bounds to error probability for coding in discrete memoryless channels. I," Information and Control, vol. 10, pp. 65-103, 1967.

[36] V. Strassen, "Asymptotische Abschätzugen in Shannon's Informationstheorie," In Transactions of the Third Prague Conference on Information Theory etc, Czechoslovak Academy of Sciences, Prague, pp. 689-723, 1962.

[37] M. Hayashi, "Information Spectrum Approach to Second-Order Coding Rate in Channel Coding," IEEE Trans. Inform. Theory, 55(11), 4947 4966, 2009.

[38] Y. Polyanskiy, H.V. Poor, and S. Verdú, "Channel coding rate in the finite blocklength regime," IEEE Trans. Inform. Theory, 56(5), 2307 $2359,2010$.

[39] S. Watanabe and M. Hayashi, "Non-asymptotic analysis of privacy amplification via Rényi entropy and inf-spectral entropy," in Proceedings of the 2013 IEEE International Symposium on Information Theory, Istanbul, Turkey, 2013, pp. 2715-2719. 